\title{
Bayesian computation for the common coefficient of variation of delta-lognormal distributions with application to common rainfall dispersion in Thailand
}

\author{
Noppadon Yosboonruang ${ }^{1}$, Sa-Aat Niwitpong ${ }^{\text {Corresp., } 1}$, Suparat Niwitpong ${ }^{1}$ \\ ${ }^{1}$ Department of Applied Statistics, King Mongkut's University of Technology North Bangkok, Bangkok, Thailand \\ Corresponding Author: Sa-Aat Niwitpong \\ Email address: sa-aat.n@sci.kmutnb.ac.th
}

Rainfall fluctuation makes precipitation and flood prediction difficult. The coefficient of variation can be used to measure rainfall dispersion to produce information for predicting future rainfall, thereby mitigating future disasters. Rainfall data usually consist of positive and true zero values that correspond to a delta-lognormal distribution. Therefore, the coefficient of variation of delta-lognormal distribution is appropriate to measure the rainfall dispersion more than lognormal distribution. In particular, the measurement of the dispersion of precipitation from several areas can be determined by measuring the common coefficient of variation in the rainfall from those areas together. Herein, we compose confidence intervals for the common coefficient of variation of delta-lognormal distributions by employing the fiducial generalized confidence interval, equal-tailed Bayesian credible intervals incorporating the independent Jeffreys or uniform priors, and the method of variance estimates recovery. A combination of the coverage probabilities and expected lengths of the proposed methods obtained via a Monte Carlo simulation study were used to compare their performances. The results show that the equal-tailed Bayesian based on the independent Jeffreys prior was suitable. In addition, it can be used the equal-tailed Bayesian based on the uniform prior as an alternative. The efficacies of the proposed confidence intervals are demonstrated via applying them to analyze daily rainfall datasets from Nan, Thailand. 


\title{
Bayesian computation for the common
} coefficient of variation of delta-lognormal distributions with application to common rainfall dispersion in Thailand

\author{
${ }_{5}$ Noppadon Yosboonruang ${ }^{1}$, Sa-Aat Niwitpong ${ }^{1}$, and Suparat Niwitpong ${ }^{1}$ \\ $6{ }^{1}$ Department of Applied Statistics, King Mongkut's University of Technology North \\ Bangkok, Bangkok, Thailand \\ 8 Corresponding author: \\ 9 Sa-Aat Niwitpong ${ }^{1}$ \\ Email address: sa-aat.n@sci.kmutnb.ac.th
}

\begin{abstract}
Rainfall fluctuation makes precipitation and flood prediction difficult. The coefficient of variation can be used to measure rainfall dispersion to produce information for predicting future rainfall, thereby mitigating future disasters. Rainfall data usually consist of positive and true zero values that correspond to a delta-lognormal distribution. Therefore, the coefficient of variation of delta-lognormal distribution is appropriate to measure the rainfall dispersion more than lognormal distribution. In particular, the measurement of the dispersion of precipitation from several areas can be determined by measuring the common coefficient of variation in the rainfall from those areas together. Herein, we compose confidence intervals for the common coefficient of variation of delta-lognormal distributions by employing the fiducial generalized confidence interval, equal-tailed Bayesian credible intervals incorporating the independent Jeffreys or uniform priors, and the method of variance estimates recovery. A combination of the coverage probabilities and expected lengths of the proposed methods obtained via a Monte Carlo simulation study were used to compare their performances. The results show that the equal-tailed Bayesian based on the independent Jeffreys prior was suitable. In addition, it can be used the equal-tailed Bayesian based on the uniform prior as an alternative. The efficacies of the proposed confidence intervals are demonstrated via applying them to analyze daily rainfall datasets from Nan, Thailand.
\end{abstract}

\section{INTRODUCTION}

Currently, the anthropomorphic emissions of greenhouse gases, sulfate aerosols, and black carbon are having a seriously deleterious effect on the Earth's climate (Nema et al., 2012). This phenomenon is directly increasing the global temperature, warming the oceans, and melting the polar ice caps, thereby causing a rise in sea level and initiating extreme weather events (NASA, 2020). Southeast Asia is a tropical area that is affected by ocean currents, prevailing winds, and abundant rainfall during the monsoon season (WorldAtlas, 2021). Thailand is located in Southeast Asia, where the climate is influenced by the monsoon winds. Especially, the combined effect of the southwest monsoon, the Inter-Tropical Convergence Zone, and tropical cyclones causes plenty of rain to fall over the country (Thai Meteorological Department, 2015). Large amounts of rainfall cause regular flooding in some areas of the country, thereby leading to damage to property and loss of life. Moreover, Thailand is an agricultural country, and rainfall fluctuation makes it difficult to predict heavy precipitation that may cause loss of or damage to crops. Therefore, it is necessary to measure the dispersion of rainfall in specific areas by using statistical tools such as the coefficient of variation $(\mathrm{CV})$ to enable accurate prediction of future catastrophic events. Nan is a province in Thailand located near the origin of the Nan River that flows into the Chao Phraya River. Furthermore, throughout the year, the precipitation in Nan fluctuates between a precipitation deficit and heavy rainfall. The latter accompanied by thunderstorms occurs in the late summer period, and due to the southwest monsoon, the amount of daily rainfall increases from mid-May to early October with the highest daily rainfall frequently 
in August or September, which can cause flooding in some areas (Thai Meteorological Department, 2015). Therefore, datasets of daily rainfall from the three areas (Chiang Klang, Tha Wang Pha, and Pua) in Nan province in August 2018 and 2019 were selected. These data comprise positive values that conform to a lognormal distribution, and true zero values, in which the frequency conforms to a binomial distribution, as presented in Fig 1. In addition, the normality plots shown in Fig 2 together with the Akaike information criterion (AIC) and the Bayesian information criterion (BIC) indicate that the daily rainfall data from the three areas follow delta-lognormal distributions. Furthermore, many researchers have reported that rainfall data follow a delta-lognormal distribution (Fukuchi, 1988; Shimizu, 1993; Yue, 2000; Kong et al., 2012; Maneerat et al., 2019a, 2020a,b; Yosboonruang et al., 2019b, 2020; Yosboonruang and Niwitpong, 2020).
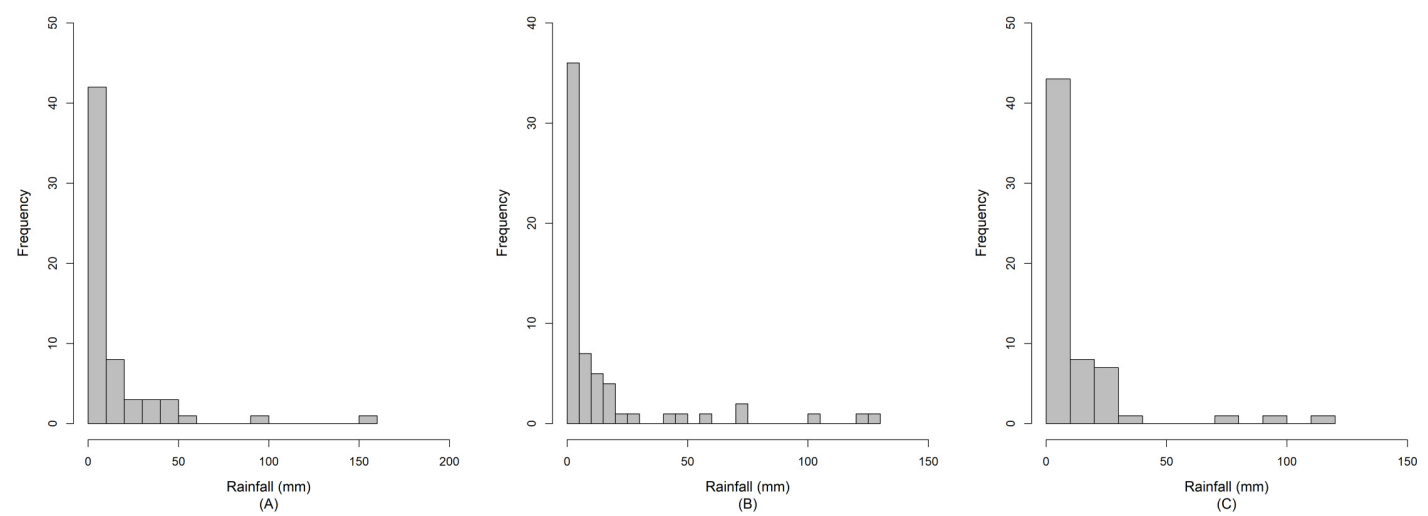

Figure 1. Histograms of the daily rainfall data from (A) Chiang Klang, (B) Tha Wang Pha, and (C) Pua in Nan, Thailand.
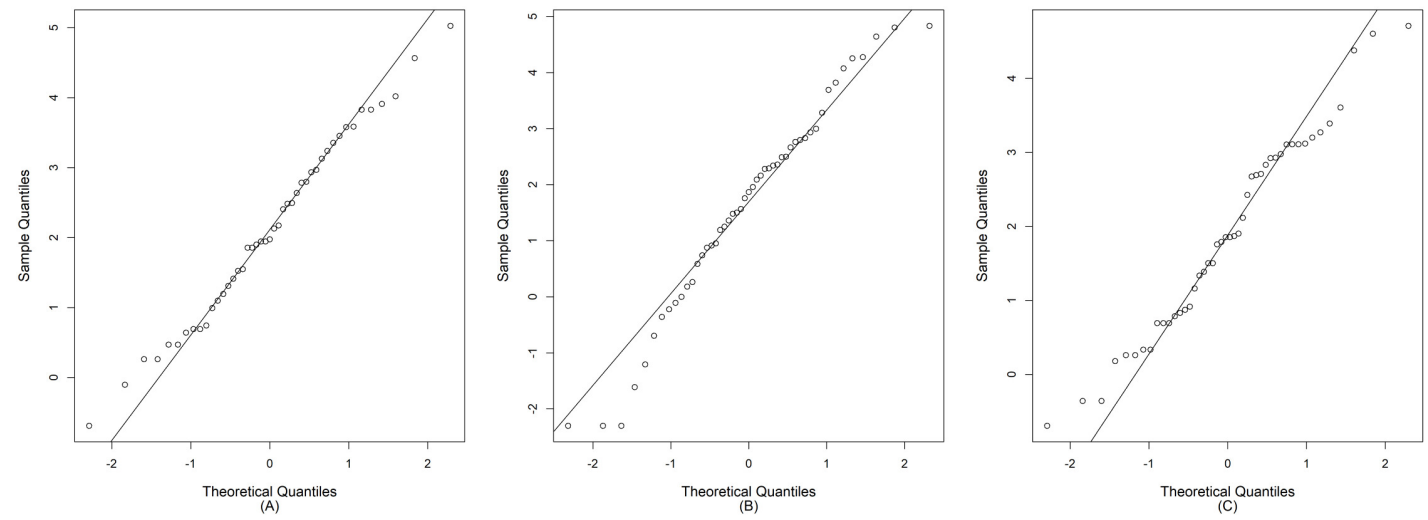

Figure 2. The normal Q-Q plots of the log-transformation of the positive daily rainfall data from (A) Chiang Klang, (B) Tha Wang Pha, and (C) Pua in Nan, Thailand.

Since the $\mathrm{CV}$ is the ratio of the mean and the standard deviation of a population, it is free from units of measurement and is often used to measure the dispersion of data and compare it between populations. For statistical inference, several methods for constructing confidence intervals for the CV and functions of the CV have been suggested (e.g. Pang et al. (2005); Hayter (2015); Nam and Kwon (2017); Yosboonruang et al. (2018, 2019a,b, 2020); Yosboonruang and Niwitpong (2020)). However, since using the common $\mathrm{CV}$ of delta-lognormal distributions for statistical inference has not previously been reported, this has become our research interest as it is useful for measuring the dispersion in several independent data series, especially rainfall data.

Several statisticians have suggested confidence intervals for the common CV of normal and non- 
Let $X_{i j}, i=1,2, \ldots, k, j=1,2, \ldots, n_{i}$ be a random variable of size $n_{i}$ from $k$ delta-lognormal distributions with density function

$$
f\left(x_{i j} ; \mu_{i}, \sigma_{i}^{2}, \delta_{i}\right)=\left(1-\delta_{i}\right) \mathrm{I}_{0}\left[x_{i j}\right]+\delta_{i} \frac{1}{x_{i j} \sqrt{2 \pi} \sigma_{i}} \exp \left\{-\frac{1}{2}\left[\frac{\ln \left(x_{i j}\right)-\mu_{i}}{\sigma_{i}}\right]^{2}\right\} \mathrm{I}_{(0, \infty)}\left[x_{i j}\right],
$$

where $\mathrm{I}_{0}\left[x_{i j}\right]$ is an indicator function for which the values are 1 when $x_{i j}=0$, and 0 otherwise; $\mathrm{I}_{(0, \infty)}\left[x_{i j}\right]$ are equal to 0 and 1 when $x_{i j}=0$ and $x_{i j}>0$, respectively; and $\delta_{i}=P\left(X_{i j}>0\right)$. This distribution is a combination of lognormal and binomial distributions. The numbers of positive and zero observations are 
defined as $n_{i 1}$ and $n_{i 0}$, respectively, where $n_{i}=n_{i 1}+n_{i 0}$. According to Aitchison (1955), the mean and variance of a delta-lognormal distribution are defined as

$$
E\left(X_{i j}\right)=\delta_{i} \exp \left(\mu_{i}+\frac{\sigma_{i}^{2}}{2}\right)
$$

and

$$
\sigma_{i}^{2}=\delta_{i} \exp \left(2 \mu_{i}+\sigma_{i}^{2}\right)\left[\exp \left(\sigma_{i}^{2}\right)-\delta_{i}\right],
$$

respectively. Since the $\mathrm{CV}$ computed from $\sigma_{i} / \mu_{i}$, then

$$
C V\left(X_{i j}\right)=\eta_{i}=\left[\frac{\exp \left(\sigma_{i}^{2}\right)-\delta_{i}}{\delta_{i}}\right]^{\frac{1}{2}}
$$

By using the log-transformation (Yosboonruang et al., 2018), let

$$
\varphi_{i}=\frac{1}{2}\left\{\ln \left[\exp \left(\sigma_{i}^{2}\right)-\delta_{i}\right]-\ln \left(\delta_{i}\right)\right\} .
$$

The unbiased estimators for $\sigma_{i}^{2}$ and $\delta_{i}$ are $\hat{\sigma}_{i}^{2}=\sum_{j=1}^{n_{i 1}}\left[\ln \left(x_{i j}\right)-\hat{\mu}_{i}\right]^{2} /\left(n_{i 1}-1\right)$ and $\hat{\delta}_{i}=n_{i 1} / n_{i}$, for $i=$ $1,2, \ldots, k$, where $\hat{\mu}_{i}=\sum_{j=1}^{n_{i 1}} \ln \left(x_{i j}\right) / n_{i 1}$, respectively, then

$$
\hat{\varphi}_{i}=\frac{1}{2}\left\{\ln \left[\exp \left(\hat{\sigma}_{i}^{2}\right)-\hat{\delta}_{i}\right]-\ln \left(\hat{\delta}_{i}\right)\right\} .
$$

The approximately unbiased estimate variance of $\hat{\varphi}_{i}$ is

$$
\hat{V}\left(\hat{\varphi}_{i}\right) \approx \frac{\left(\hat{b}_{i}-\hat{a}_{i}\right)\left(1-\hat{a}_{i} \hat{b}_{i}\right)-n_{i 1}\left(1-\hat{a}_{i}\right)^{2}}{4 n_{i 1}\left(1-\hat{a}_{i}\right)^{2}}+\frac{\hat{\sigma}_{i}^{4}}{2\left(n_{i 1}-1\right)},
$$

where $\hat{a}_{i}=\left(1-\hat{\delta}_{i}\right)^{n_{i}-1}$ and $\hat{b}_{i}=1+\left(n_{i}-1\right) \hat{\delta}_{i}$. The ordinary form of the common log-transformed CV is given by

$$
\tilde{\varphi}=\frac{\sum_{i=1}^{k} w_{i} \hat{\varphi}_{i}}{\sum_{i=1}^{k} w_{i}}
$$

where $w_{i}=1 / \hat{V}\left(\hat{\varphi}_{i}\right)$. Accordingly, the common $\mathrm{CV}$ is defined as

$$
\tilde{\eta}=\exp (\tilde{\varphi})=\exp \left(\frac{\sum_{i=1}^{k} w_{i} \hat{\varphi}_{i}}{\sum_{i=1}^{k} w_{i}}\right) .
$$

Here, the methods to establish the confidence intervals for the common CV for delta-lognormal distributions are provided in detail.

\section{FGCI}

Let $X_{i j}, i=1,2, \ldots, k, j=1,2, \ldots, n_{i}$ be a random sample with density function $f\left(x_{i j} ; \theta_{i}, \mu_{i}\right)$, where $\theta_{i}=\left(\delta_{i}, \sigma_{i}^{2}\right)$ are the parameters of interest and $\mu_{i}$ is a nuisance parameter. Let $x_{i j}$ be the observed values of $X_{i j}$. To construct the FGCI (Weerahandi, 1993; Hannig et al., 2006), the fiducial generalized pivotal quantity (FGPQ) $R\left(X_{i j} ; x_{i j}, \theta_{i}, \mu_{i}\right)$ is needed to satisfy the following two properties:

1. For each $x_{i j}$, the conditional distribution of $R\left(X_{i j} ; x_{i j}, \theta_{i}, \mu_{i}\right)$ is unaffected by the nuisance parameter.

2. The observed value of $R\left(X_{i j} ; x_{i j}, \theta_{i}, \mu_{i}\right), r\left(x_{i j} ; x_{i j}, \theta_{i}, \mu_{i}\right)$, is the parameter of interest.

Given that $R_{\alpha}$ is the $100 \alpha$ - th percentile of $R\left(X_{i j} ; x_{i j}, \theta_{i}, \mu_{i}\right)$, then $\left(R_{\alpha / 2}, R_{1-\alpha / 2}\right)$ becomes the $100(1-\alpha) \%$ two-sided FGCI for $\theta_{i}$. Hence, it is essential to use the FGPQs for $\delta_{i}$ and $\sigma_{i}^{2}$ to construct the confidence interval for the common $\mathrm{CV}(\tilde{\eta})$ for delta-lognormal distributions. 
Consider $k$ individual random samples $X_{i 1}, X_{i 2}, \ldots, X_{i n_{i}}$. Following Hannig (2009) and Li et al. (2013), the FGPQ for $\delta_{i}$ is as follows

$$
R_{\delta_{i}} \sim \frac{1}{2} \operatorname{Beta}\left(n_{i 1}, n_{i 0}+1\right)+\frac{1}{2} \operatorname{Beta}\left(n_{i 1}+1, n_{i 0}\right)
$$

Similarly, Wu and Hsieh (2014) followed the concept of Krishnamoorthy and Mathew (2003) to find the FGPQ for $\sigma_{i}^{2}$ defined as

$$
R_{\sigma_{i}^{2}}=\frac{\left(n_{i 1}-1\right) \hat{\sigma}_{i}^{2}}{U_{i}}
$$

where $U_{i} \sim \chi_{n_{i 1}-1}^{2}$. To find the FGPQ for $\hat{\varphi}$, we then substitute $R_{\delta_{i}}$ and $R_{\sigma_{i}^{2}}$ into Eq (6) as follows:

$$
R_{\hat{\varphi}_{i}}=\frac{1}{2}\left\{\ln \left[\exp \left(R_{\sigma_{i}^{2}}\right)-\ln \left(R_{\delta_{i}}\right)\right]-\ln \left(R_{\delta_{i}}\right)\right\} .
$$

Consequently, the FGPQ for common $\mathrm{CV}(\tilde{\eta})$ is

$$
R_{\tilde{\eta}}=\exp \left(\frac{\sum_{i=1}^{k} R_{w_{i}} R_{\hat{\varphi}_{i}}}{\sum_{i=1}^{k} R_{w_{i}}}\right)
$$

where the FGPQ for an estimated variance of $\hat{\varphi}_{i}$, for which $R_{w_{i}}$ is the inverse, is given by

$$
R_{\hat{V}\left(\hat{\varphi}_{i}\right)}=\frac{\left(R_{b_{i}}-R_{a_{i}}\right)\left(1-R_{a_{i}} R_{b_{i}}\right)-n_{i 1}\left(1-R_{a_{i}}\right)^{2}}{4 n_{i 1}\left(1-R_{a_{i}}\right)^{2}}+\frac{R_{\sigma_{i}^{2}}^{2}}{2\left(n_{i 1}-1\right)},
$$

where $R_{a_{i}}=\left(1-R_{\delta_{i}}\right)^{n_{i}-1}$ and $R_{b_{i}}=1+\left(n_{i}-1\right) R_{\delta_{i}}$.

Thus, we employ $R_{\tilde{\eta}}$ to produce the confidence interval for $\tilde{\eta}$. Consequently, the $100(1-\alpha) \%$ two-sided confidence interval for $\tilde{\eta}$ based on FGCI becomes $\left(R_{\tilde{\eta}}(\alpha / 2), R_{\tilde{\eta}}(1-\alpha / 2)\right)$, which denote the $\alpha / 2$ th and $(1-\alpha / 2)$ th percentiles of $R_{\tilde{\eta}}$, respectively.

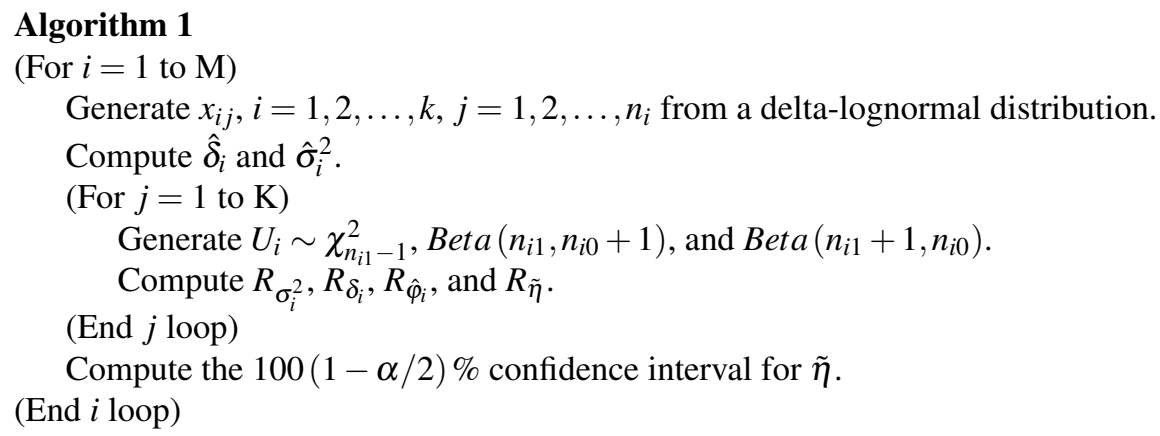

\section{Bayesian methods}

Since random samples $X_{i j}, i=1,2, \ldots, k, j=1,2, \ldots, n_{i}$ have a delta-lognormal distribution with unknown parameters $\phi=\left(\delta_{i}^{*}, \mu_{i}, \sigma_{i}^{2}\right)$, where $\delta_{i}^{*}=1-\delta_{i}$, the likelihood function of $k$-individual random samples can be expressed as

$$
L\left(\phi \mid x_{i j}\right) \propto \prod_{i=1}^{k}\left(\delta_{i}^{*}\right)^{n_{i 0}} \delta_{i}^{n_{i 1}}\left(\sigma_{i}^{2}\right)^{-\frac{n_{i 1}}{2}} \exp \left\{-\frac{1}{2 \sigma_{i}^{2}} \sum_{j=1}^{n_{i 1}}\left[\ln \left(x_{i j}\right)-\mu_{i}\right]^{2}\right\} .
$$

Subsequently, the Fisher information matrix of $\phi$ based on the partial derivatives of the log-likelihood functions for $\delta_{i}, \mu_{i}$, and $\sigma_{i}^{2}$ becomes

$$
I(\phi)=\operatorname{diag}\left[\begin{array}{lllllllll}
\frac{n_{1}}{\delta_{1}^{*} \delta_{1}} & \frac{n_{1} \delta_{1}}{\sigma_{1}^{2}} & \frac{n_{1} \delta_{1}}{2\left(\sigma_{1}^{2}\right)^{2}} & \cdots & \cdots & \cdots & \frac{n_{k}}{\delta_{k}^{*} \delta_{k}} & \frac{n_{k} \delta_{k}}{\sigma_{k}^{2}} & \frac{n_{k} \delta_{k}}{2\left(\sigma_{k}^{2}\right)^{2}}
\end{array}\right] .
$$

In the present study, the Bayesian method is used to construct the equal-tailed confidence interval and the credible interval for the common CV. In the following section, we propose the independent Jeffreys and uniform priors. 


\section{The Bayesian method using the independent Jeffreys prior}

Since Jeffreys' prior for unknown parameter $\phi$ is derived from the square root of the determinant of Fisher information matrix $I(\phi)$, then $p(\phi)=\sqrt{|I(\phi)|}$. Since the parameters of interest $\vartheta=\left(\delta_{i}^{*}, \sigma_{i}^{2}\right)$, the independent Jeffreys prior for $\delta_{i}$ and $\sigma_{i}^{2}$ are $p\left(\delta_{i}\right) \propto\left(\delta_{i}^{*}\right)^{-\frac{1}{2}} \delta_{i}^{-\frac{1}{2}}$ and $p\left(\sigma_{i}^{2}\right) \propto 1 / \sigma_{i}^{2}$ (Harvey and van der Merwe, 2012), respectively. Since $\delta_{i}^{*}$ and $\sigma_{i}^{2}$ are independent, then the independent Jeffreys prior for a delta-lognormal distribution is $p(\vartheta) \propto \prod_{i=1}^{k} \sigma_{i}^{-2}\left(\delta_{i}^{*}\right)^{-\frac{1}{2}} \delta_{i}^{-\frac{1}{2}}$. Thus, the joint posterior density of $\phi$ becomes

$$
\begin{aligned}
p\left(\phi \mid x_{i j}\right)= & \prod_{i=1}^{k} \frac{1}{\operatorname{Beta}\left(n_{i 0}+\frac{1}{2}, n_{i 1}+\frac{1}{2}\right)}\left(\delta_{i}^{*}\right)^{\left(n_{i 0}+\frac{1}{2}\right)-1} \delta_{i}^{\left(n_{i 1}+\frac{1}{2}\right)-1} \frac{1}{\sqrt{2 \pi} \frac{\sigma_{i}}{\sqrt{n_{i 1}}}} \exp \left[-\frac{1}{2 \frac{\sigma_{i}^{2}}{n_{i 1}}}\left(\mu_{i}-\hat{\mu}_{i}\right)^{2}\right] \\
& \times \frac{\left[\frac{\left(n_{i 1}-1\right) \hat{\sigma}_{i}^{2}}{2}\right]^{\frac{n_{i 1}-1}{2}}}{\Gamma\left(\frac{n_{i 1}-1}{2}\right)}\left(\sigma_{i}^{2}\right)^{-\frac{n_{i 1}-1}{2}-1} \exp \left[-\frac{\frac{\left(n_{i 1}-1\right) \hat{\sigma}_{i}^{2}}{2}}{\sigma_{i}^{2}}\right]
\end{aligned}
$$

where $\hat{\mu}_{i}=\sum_{j=1}^{n_{i 1}} \ln \left(x_{i j}\right) / n_{i 1}$, and $\hat{\sigma}_{i}^{2}=\sum_{j=1}^{n_{i 1}}\left[\ln \left(x_{i j}\right)-\hat{\mu}_{i}\right]^{2} /\left(n_{i 1}-1\right)$. This leads to the posterior density of $\delta_{i}^{*}$ given by

$$
p\left(\delta_{i}^{*} \mid x_{i j}\right) \propto \prod_{i=1}^{k} \frac{1}{\operatorname{Beta}\left(n_{i 0}+\frac{1}{2}, n_{i 1}+\frac{1}{2}\right)}\left(\delta_{i}^{*}\right)^{\left(n_{i 0}+\frac{1}{2}\right)-1} \delta_{i}^{\left(n_{i 1}+\frac{1}{2}\right)-1},
$$

which is a beta distribution with parameters $n_{i 0}+1 / 2$ and $n_{i 1}+1 / 2$, denoted by $\delta_{i}^{*} \mid x_{i j} \sim \operatorname{Beta}\left(n_{i 0}+1 / 2, n_{i 1}+1 / 2\right)$. Similarly, the posterior density of $\sigma_{i}^{2}$ can be derived as

$$
p\left(\sigma_{i}^{2} \mid x_{i j}\right) \propto \frac{\left[\frac{\left(n_{i 1}-1\right) \hat{\sigma}_{i}^{2}}{2}\right]^{\frac{n_{i 1}-1}{2}}}{\Gamma\left(\frac{n_{i 1}-1}{2}\right)}\left(\sigma_{i}^{2}\right)^{-\frac{n_{i 1}-1}{2}-1} \exp \left[-\frac{\frac{\left(n_{i 1}-1\right) \hat{\sigma}_{i}^{2}}{2}}{\sigma_{i}^{2}}\right],
$$

which is in the general form of an inverse gamma distribution denoted by $\sigma_{i}^{2} \mid x_{i j} \sim \operatorname{Inv}-\operatorname{Gamma}\left[\left(n_{i 1}-1\right) / 2,\left(n_{i 1}-1\right) \hat{\sigma}_{i}^{2} / 2\right]$.

\section{The Bayesian method using the uniform prior}

Because all possible values are equally likely a priori for the uniform prior, then it is a constant function of a priori probability (Stone, 2013; O'Reilly and Mars, 2015). According to Bolstad and Curran (2016), the uniform prior for $\delta_{i}^{*}$ and $\sigma_{i}^{2}$ are proportional to 1 , which can be defined as $p\left(\delta_{i}^{*}\right) \propto 1$ and $p\left(\sigma_{i}^{2}\right) \propto 1$, respectively. It is well-known that $\delta_{i}^{*}$ is independent of $\sigma_{i}^{2}$, thereby the uniform prior for the parameters of interest for a delta-lognormal distribution is $p\left(\delta_{i}^{*}, \sigma_{i}^{2}\right) \propto 1$. Accordingly, the joint posterior density function is defined as

$$
\begin{aligned}
p\left(\phi \mid x_{i j}\right)= & \prod_{i=1}^{k} \frac{1}{\operatorname{Beta}\left(n_{i 0}+1, n_{i 1}+1\right)}\left(\delta_{i}^{*}\right)^{n_{i 0}} \delta_{i}^{n_{i 1}} \frac{1}{\sqrt{2 \pi} \frac{\sigma_{i}}{\sqrt{n_{i 1}}}} \exp \left[-\frac{1}{2 \frac{\sigma_{i}^{2}}{n_{i 1}}}\left(\mu_{i}-\hat{\mu}_{i}\right)^{2}\right] \\
& \times \frac{\left[\frac{\left(n_{i 1}-2\right) \hat{\sigma}_{i}^{2}}{2}\right]^{\frac{n_{i 1}-2}{2}}}{\Gamma\left(\frac{n_{i 1}-2}{2}\right)}\left(\sigma_{i}^{2}\right)^{-\frac{n_{i 1}-2}{2}-1} \exp \left[-\frac{\frac{\left(n_{i 1}-2\right) \hat{\sigma}_{i}^{2}}{2}}{\sigma_{i}^{2}}\right],
\end{aligned}
$$

where $\hat{\mu}_{i}=\sum_{j=1}^{n_{i 1}} \ln \left(x_{i j}\right) / n_{i 1}$, and $\hat{\sigma}_{i}^{2}=\sum_{j=1}^{n_{i 1}}\left[\ln \left(x_{i j}\right)-\hat{\mu}_{i}\right]^{2} /\left(n_{i 1}-1\right)$. We can derived the posterior density of $\delta_{i}^{*}$ as

$$
p\left(\delta_{i}^{*} \mid x_{i j}\right) \propto \prod_{i=1}^{k} \frac{1}{\operatorname{Beta}\left(n_{i 0}+1, n_{i 1}+1\right)}\left(\delta_{i}^{*}\right)^{n_{i 0}} \delta_{i}^{n_{i 1}},
$$

which is consequently a density function of a beta distribution, i.e. $\delta_{i}^{*} \mid x_{i j} \sim \operatorname{Beta}\left(n_{i 0}+1, n_{i 1}+1\right)$. For $\sigma_{i}^{2}$, the posterior density has an inverse gamma distribution with respective shape and scale parameters 
$\left(n_{i 1}-2\right) / 2$ and $\left(n_{i 1}-2\right) \hat{\sigma}_{i}^{2} / 2$ which expressed as

$$
p\left(\sigma_{i}^{2} \mid x_{i j}\right) \propto \prod_{i=1}^{k} \frac{\left(\frac{\left(n_{i 1}-2\right) \hat{\sigma}_{i}^{2}}{2}\right)^{\frac{n_{i 1}-2}{2}}}{\Gamma\left(\frac{n_{i 1}-2}{2}\right)}\left(\sigma_{i}^{2}\right)^{-\frac{n_{i 1}-2}{2}-1} \exp \left(-\frac{\frac{\left(n_{i 1}-2\right) \hat{\sigma}_{i}^{2}}{2}}{\sigma_{i}^{2}}\right) .
$$

Subsequently, we construct the confidence intervals and the credible intervals for the common CV by substituting the posterior densities of $\delta_{i}^{*}$ and $\sigma_{i}^{2}$ from the independent Jeffreys and uniform priors into Eqs (6), (7), and (9).

\section{Algorithm 2}

(For $i=1$ to $\mathrm{M}$ )

Generate $x_{i j}, i=1,2, \ldots, k, j=1,2, \ldots, n_{i}$ from a delta-lognormal distribution.

Compute $\hat{\delta}_{i}$ and $\hat{\sigma}_{i}^{2}$.

(For $j=1$ to $\mathrm{K}$ )

Generate the posterior densities of $\delta_{i}^{*} \mid x_{i j}$.

1. Independent Jeffreys prior: $\delta_{i}^{*} \mid x_{i j} \sim \operatorname{Bet} a\left(n_{i 0}+\frac{1}{2}, n_{i 1}+\frac{1}{2}\right)$.

2. Uniform prior: $\delta_{i}^{*} \mid x_{i j} \sim \operatorname{Beta}\left(n_{i 0}+1, n_{i 1}+1\right)$.

Generate the posterior densities of $\sigma_{i}^{2} \mid x_{i j}$.

1. Independent Jeffreys prior: $\sigma_{i}^{2} \mid x_{i j} \sim$ Inv - Gamma $\left[\frac{n_{i 1}-1}{2}, \frac{\left(n_{i 1}-1\right) \hat{\sigma}_{i}^{2}}{2}\right]$.

2. Uniform prior: $\sigma_{i}^{2} \mid x_{i j} \sim$ Inv-Gamma $\left[\frac{n_{i 1}-2}{2}, \frac{\left(n_{i 1}-2\right) \hat{\sigma}_{i}^{2}}{2}\right]$.

Compute $\hat{\varphi}_{i}, \hat{V}\left(\hat{\varphi}_{i}\right)$, and $\tilde{\eta}$.

(End $j$ loop)

Compute the $100(1-\alpha / 2) \%$ confidence intervals and credible intervals for $\tilde{\eta}$.

(End $i$ loop)

\section{MOVER}

Following the method of Zou and Donner (2008), let $\varphi_{1}$ and $\varphi_{2}$ be the parameter of interest and then let $\hat{\varphi}_{1}$ and $\hat{\varphi}_{2}$ be the independent estimators of $\varphi_{1}$ and $\varphi_{2}$, respectively. Furthermore, the lower and upper confidence limits for $\varphi_{1}+\varphi_{2}$ are

$$
C I_{\varphi_{1}+\varphi_{2}}=\left[L_{\varphi_{1}+\varphi_{2}}, U_{\varphi_{1}+\varphi_{2}}\right]=\hat{\varphi}_{1}+\hat{\varphi}_{2} \pm z_{\alpha / 2} \sqrt{\widehat{\operatorname{Var}}\left(\hat{\varphi}_{1}\right)+\widehat{\operatorname{Var}}\left(\hat{\varphi}_{2}\right)},
$$

Subsequently, let $l_{i}$ and $u_{i}$, for $i=1,2$, be the lower and upper bounds of the confidence interval for $\varphi_{i}$, respectively. Since $l_{i}$ and $u_{i}$ provide the possible parameter values, then $l_{1}+l_{2}$ is close to $L_{\varphi_{1}+\varphi_{2}}$ and $u_{1}+u_{2}$ is close to $U_{\varphi_{1}+\varphi_{2}}$. To obtain the lower limit $L_{\varphi_{1}+\varphi_{2}}$, the estimated variance of $\hat{\varphi}_{i}$ at $\varphi_{i}=l_{i}$ is given by

$$
\widehat{\operatorname{Var}}\left(\hat{\varphi}_{l_{i}}\right)=\frac{\left(\hat{\varphi}_{i}-l_{i}\right)^{2}}{z_{\alpha / 2}^{2}} .
$$

Similarly, to obtain the upper limit $U_{\varphi_{1}+\varphi_{2}}$, the estimated variance of $\hat{\varphi}_{i}$ at $\varphi_{i}=u_{i}$ is given by

$$
\widehat{\operatorname{Var}}\left(\hat{\varphi}_{u_{i}}\right)=\frac{\left(u_{i}-\hat{\varphi}_{i}\right)^{2}}{z_{\alpha / 2}^{2}}
$$

Next, by substituting $\widehat{\operatorname{Var}}\left(\hat{\varphi}_{l_{i}}\right)$ and $\widehat{\operatorname{Var}}\left(\hat{\varphi}_{u_{i}}\right)$ into Eq (23), we obtain

$$
L_{\varphi_{1}+\varphi_{2}}=\hat{\varphi}_{1}+\hat{\varphi}_{2}-\sqrt{\left(\hat{\varphi}_{1}-l_{1}\right)^{2}+\left(\hat{\varphi}_{2}-l_{2}\right)^{2}}
$$

and

$$
U_{\varphi_{1}+\varphi_{2}}=\hat{\varphi}_{1}+\hat{\varphi}_{2}-\sqrt{\left(u_{1}-\hat{\varphi}_{1}\right)^{2}+\left(u_{2}-\hat{\varphi}_{2}\right)^{2}} .
$$


Thereby, the unbiased estimate variance of $\hat{\varphi}_{i}$ at $\varphi_{i}=l_{i}$ and $\varphi_{i}=u_{i}$ can be expressed as

$$
\widehat{\operatorname{Var}}\left(\hat{\varphi}_{i}\right)=\frac{1}{2}\left[\frac{\left(\hat{\varphi}_{i}-l_{i}\right)^{2}}{z_{\alpha / 2}^{2}}+\frac{\left(u_{i}-\hat{\varphi}_{i}\right)^{2}}{z_{\alpha / 2}^{2}}\right], i=1,2 .
$$

When this concept is extended to $k$ parameters, the lower and upper confidence limits for $v=\sum_{i=1}^{k} \varphi_{i}$ are given by

$$
L_{v}=v-\sqrt{\left(\hat{\varphi}_{1}-l_{1}\right)^{2}+\left(\hat{\varphi}_{2}-l_{2}\right)^{2}+\ldots+\left(\hat{\varphi}_{k}-l_{k}\right)^{2}}
$$

and

$$
U_{v}=v+\sqrt{\left(u_{1}-\hat{\varphi}_{1}\right)^{2}+\left(u_{2}-\hat{\varphi}_{2}\right)^{2}+\ldots+\left(u_{k}-\hat{\varphi}_{k}\right)^{2}} .
$$

According to Krishnamoorthy and Oral (2017) and recall the common log-transformed CV from Eq (8), the upper and lower confidence limits for $\sigma_{i}^{2}$ and $\delta_{i}$ are required to construct the confidence interval for the common $\mathrm{CV}$ of delta-lognormal distributions. Since the estimate of $\sigma_{i}^{2}$ is

$$
\hat{\sigma}_{i}^{2}=\frac{1}{n_{i 1}-1} \sum_{j=1}^{n_{i 1}}\left[\ln \left(x_{i j}\right)-\hat{\mu}_{i}\right]^{2},
$$

where $\left(n_{i 1}-1\right) \hat{\sigma}_{i}^{2} / \sigma_{i}^{2} \sim \chi_{n_{i 1}-1}^{2}$, the $100(1-\alpha) \%$ confidence interval for $\sigma_{i}^{2}$ is derived as

$$
C I_{\sigma_{i}^{2}}=\left(l_{\sigma_{i}^{2}}, u_{\sigma_{i}^{2}}\right)=\left[\frac{\left(n_{i 1}-1\right) \hat{\sigma}_{i}^{2}}{\chi_{1-\alpha / 2, n_{i 1}-1}^{2}}, \frac{\left(n_{i 1}-1\right) \hat{\sigma}_{i}^{2}}{\chi_{\alpha / 2, n_{i 1}-1}^{2}}\right]
$$

To construct the confidence interval for $\delta_{i}$, the concept of the variance stabilizing transformation proposed by DasGupta (2008) and Wu and Hsieh (2014) was used. Therefore, the confidence interval for $\delta_{i}$ is given by

$$
C I_{\delta_{i}}=\left(l_{\delta_{i}}, u_{\delta_{i}}\right)=\sin ^{2}\left(\arcsin \sqrt{\hat{\delta}_{i}} \pm \frac{1}{2 \sqrt{n_{i}}} Z_{i(1-\alpha / 2)}\right)
$$

Since $\varphi_{i}=\left\{\ln \left[\exp \left(\sigma_{i}^{2}\right)-\delta_{i}\right]-\ln \left(\delta_{i}\right)\right\} / 2$, then let

$$
l_{i}=\frac{1}{2}\left\{\ln \left[\exp \left(l_{\sigma_{i}^{2}}\right)-l_{\delta_{i}}\right]-\ln \left(l_{\delta_{i}}\right)\right\}
$$

and

$$
u_{i}=\frac{1}{2}\left\{\ln \left[\exp \left(u_{\sigma_{i}^{2}}\right)-u_{\delta_{i}}\right]-\ln \left(u_{\delta_{i}}\right)\right\}
$$

for $i=1,2, \ldots, k$. Therefore, the $100(1-\alpha) \%$ confidence interval for $\tilde{\eta}$ based on MOVER can be written as

$$
C I_{\tilde{\eta}}^{m}=\left[L_{\tilde{\eta}}^{m}, U_{\tilde{\eta}}^{m}\right]
$$

where

$$
L_{\tilde{\eta}}^{m}=\exp \left(\tilde{\varphi}-\sqrt{\sum_{i=1}^{k} w_{i}^{2}\left(\hat{\varphi}_{i}-l_{i}\right)^{2} / \sum_{i=1}^{k} w_{i}^{2}}\right)
$$

and

$$
U_{\tilde{\eta}}^{m}=\exp \left(\tilde{\varphi}+\sqrt{\sum_{i=1}^{k} w_{i}^{2}\left(u_{i}-\hat{\varphi}_{i}\right)^{2} / \sum_{i=1}^{k} w_{i}^{2}}\right) .
$$




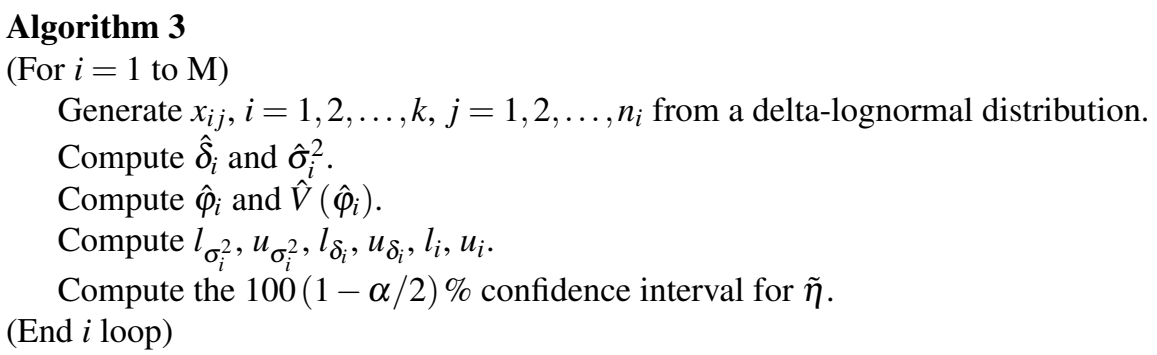

\section{RESULTS}

\section{Monte Carlo simulation studies}

The R statistical program was used to run the Monte Carlo simulation study and calculate the results for evaluating the performances of FGCI, MOVER, and the Bayesian intervals with the independent Jeffreys or uniform priors. The criteria for choosing the best performing confidence interval were coverage probability $\geq 0.95$ and the shortest expected length for each scenario tested. To generate the data, we set the number of populations as $k=3,5,10$; sample sizes as $n_{1}=n_{2}=\ldots=n_{k}=n=25,50,100$; probabilities of non-zero values as $\delta_{1}=\delta_{2}=\ldots=\delta_{k}=\delta=0.2,0.5,0.8$; and variances as $\sigma_{1}^{2}=\sigma_{2}^{2}=$ $\ldots=\sigma_{k}^{2}=\sigma^{2}=0.1,0.5,1.0,2.0$. For each combination of parameters, 10,000 simulation runs were generated together with 2,000 replications for FGCI and the Bayesian approaches by applying Algorithms 1 and 2 , respectively.

The result for the $95 \%$ confidence and credible intervals for the common $\mathrm{CV}$ of delta-lognormal distributions for various sample sizes, probabilities of non-zero values, and variances are reported in Tables 1 - 3 and displayed in Figs 3 - 8. The equal-tailed Bayesian credible intervals based on the independent Jeffreys or uniform priors produced coverage probabilities close to or greater than the nominal confidence level for almost all of the scenarios whereas the others could achieve this in only some of them. Furthermore, in terms of the expected lengths, the equal-tailed based on independent Jeffreys prior were shorter than the uniform prior for all cases. In addition, the expected lengths of the Bayesian credible interval based on the independent Jeffreys prior were shorter than the others in almost every case when $\sigma_{i}^{2}=0.5$. For all $k$ and sample sizes together with $\sigma_{i}^{2}=1,2$, the expected lengths of the equal-tailed Bayesian based on the independent Jeffreys prior were the shortest when $\delta_{i}=0.2,0.5$, while MOVER had the shortest expected lengths for $\delta_{i}=0.8$. For FGCI, the coverage probabilities and their expected lengths were very wide for all cases in which it is not reasonable for the construction of confidence interval. However, the equal-tailed Bayesian interval based on the independent Jeffreys prior can be used to derive the confidence interval for the common $\mathrm{CV}$ of delta-lognormal distributions since it produced coverage probabilities $\geq 0.95$ for almost all cases, although the expected lengths were not always shorter than the other methods in some cases.

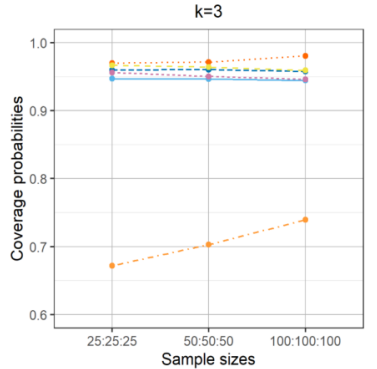

(A)

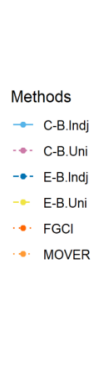

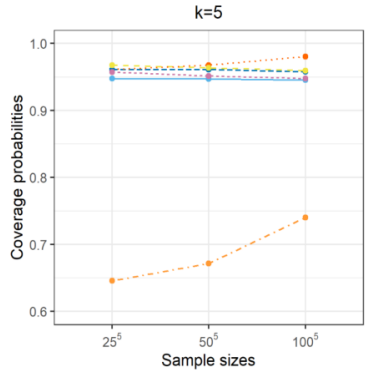

(B)
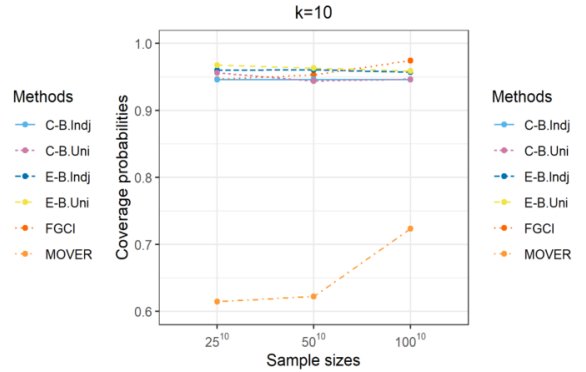

(C)

Figure 3. Comparison of the coverage probabilities of the proposed methods according to sample sizes for (A) $k=3$ (B) $k=5$ (C) $k=10$.

\section{Application of the methods to real datasets}

Datasets of daily rainfall from Chiang Klang, Tha Wang Pha, and Pua in Nan, Thailand, were obtained from the Upper Northern Region Irrigation Hydrology Center. The reason for using these datasets is 
Table 1. The results for the $95 \%$ two-sided confidence intervals for the common CV of delta-lognormal distributions for $k=3$.

\begin{tabular}{|c|c|c|c|c|c|c|c|c|}
\hline \multirow[b]{2}{*}{$n_{i}$} & \multirow{2}{*}{$\delta_{i}$} & \multirow{2}{*}{$\sigma_{i}^{2}$} & \multicolumn{6}{|c|}{ Coverage probabilities (Expected lengths) } \\
\hline & & & FGCI & E-B.Indj & E-B.Uni & C-B.Indj & C-B.Uni & MOVER \\
\hline \multirow[t]{16}{*}{25} & \multirow[t]{8}{*}{0.5} & 0.1 & 0.9740 & $\mathbf{0 . 9 5 3 7}$ & 0.9601 & 0.9357 & 0.9411 & 0.2536 \\
\hline & & & $(0.7272)$ & $(0.7291)$ & $(0.7249)$ & $(0.7079)$ & $(0.7037)$ & $(0.3519)$ \\
\hline & & 0.5 & 0.9983 & 0.9689 & 0.9734 & 0.9518 & 0.9564 & 0.5541 \\
\hline & & & $(4.2363)$ & $(1.5221)$ & $(1.6132)$ & (1.3548) & $(1.4144)$ & $(0.4478)$ \\
\hline & & 1.0 & 0.9775 & 0.9613 & 0.9672 & 0.9429 & 0.9518 & 0.7872 \\
\hline & & & $(28.1984)$ & $(4.2206)$ & $(4.7049)$ & $(3.2428)$ & (3.5018) & $(1.4839)$ \\
\hline & & 2.0 & 0.9027 & 0.9528 & 0.9600 & 0.9408 & 0.9485 & 0.9133 \\
\hline & & & (7.4009) & $(\mathbf{3 4 . 3 8 4 6 )}$ & $(42.9115)$ & $(18.1504)$ & $(20.8831)$ & $(8.0816)$ \\
\hline & \multirow[t]{8}{*}{0.8} & 0.1 & 0.9959 & 0.9676 & 0.9823 & 0.9479 & 0.9699 & 0.1611 \\
\hline & & & $(0.5921)$ & $(0.4309)$ & $(0.4381)$ & $(0.4188)$ & $(0.4263)$ & $(0.1540)$ \\
\hline & & 0.5 & 0.9994 & 0.9658 & 0.9700 & 0.9575 & 0.9687 & 0.7894 \\
\hline & & & $(9.1319)$ & $(0.8848)$ & $(0.9205)$ & $(0.8193)$ & $(0.8486)$ & $(0.3732)$ \\
\hline & & 1.0 & 0.9890 & 0.9563 & 0.9623 & 0.9526 & 0.9589 & 0.9445 \\
\hline & & & (274.3619) & $(2.0822)$ & (2.1950) & (1.7915) & (1.8716) & $(1.1456)$ \\
\hline & & 2.0 & 0.9204 & 0.9518 & 0.9567 & 0.9464 & 0.9548 & 0.9705 \\
\hline & & & (13.9706) & $(9.4530)$ & $(10.2449)$ & (6.8908) & (7.3329) & $(4.7949)$ \\
\hline \multirow[t]{24}{*}{50} & \multirow[t]{8}{*}{0.2} & 0.1 & 0.9510 & 0.9611 & 0.9554 & 0.9424 & 0.9346 & 0.5551 \\
\hline & & & (1.2188) & (1.3347) & (1.3067) & $(1.2849)$ & (1.2573) & $(0.7675)$ \\
\hline & & 0.5 & 0.9940 & 0.9734 & 0.9721 & 0.9589 & 0.9580 & 0.4001 \\
\hline & & & (5.3666) & (3.0387) & (3.3163) & $(2.5773)$ & (2.7137) & $(0.7416)$ \\
\hline & & 1.0 & 0.9668 & 0.9663 & $\mathbf{0 . 9 7 0 0}$ & 0.9436 & 0.9473 & 0.6903 \\
\hline & & & (61.6887) & $(10.2196)$ & (12.7965) & $(6.8382)$ & (7.7720) & $(2.3985)$ \\
\hline & & 2.0 & 0.8941 & 0.9573 & 0.9674 & 0.9403 & 0.9473 & 0.8591 \\
\hline & & & $(10.2166)$ & (205.9432) & (470.3159) & (61.6888) & $(89.7961)$ & (16.4537) \\
\hline & \multirow[t]{8}{*}{0.5} & 0.1 & 0.9746 & 0.9573 & 0.9592 & 0.9454 & 0.9463 & 0.7691 \\
\hline & & & $(0.4985)$ & $(0.5278)$ & $(0.5246)$ & $(0.5178)$ & $(0.5146)$ & $(0.3383)$ \\
\hline & & 0.5 & 0.9991 & 0.9665 & 0.9689 & 0.9517 & 0.9533 & 0.4400 \\
\hline & & & $(2.3261)$ & $(0.9184)$ & $(0.9322)$ & $(0.8743)$ & $(0.8851)$ & $(0.2156)$ \\
\hline & & 1.0 & 0.9977 & 0.9554 & 0.9608 & 0.9435 & 0.9462 & 0.7862 \\
\hline & & & (27.7099) & $(2.0097)$ & $(2.0670)$ & $(1.8046)$ & (1.8466) & $(0.7810)$ \\
\hline & & 2.0 & 0.9383 & 0.9498 & 0.9545 & 0.9385 & 0.9434 & 0.9348 \\
\hline & & & $(24.2678)$ & $(8.0467)$ & (8.3999) & $(6.4058)$ & $(6.6170)$ & (3.8741) \\
\hline & \multirow[t]{8}{*}{0.8} & 0.1 & 0.9960 & 0.9675 & 0.9766 & 0.9514 & 0.9658 & 0.3313 \\
\hline & & & $(0.3738)$ & $(0.3069)$ & $(0.3088)$ & $(0.3013)$ & $(0.3033)$ & $(0.1317)$ \\
\hline & & 0.5 & 0.9997 & $\mathbf{0 . 9 6 0 7}$ & 0.9665 & $\mathbf{0 . 9 5 0 7}$ & 0.9593 & 0.7357 \\
\hline & & & $(1.6860)$ & $(0.5521)$ & $(0.5606)$ & $(0.5316)$ & $(0.5397)$ & $(0.2003)$ \\
\hline & & 1.0 & 0.9980 & 0.9563 & 0.9584 & 0.9458 & 0.9513 & 0.9516 \\
\hline & & & (5.1781) & (1.1695) & (1.1911) & (1.0905) & (1.1088) & $(\mathbf{0 . 7 0 5 8})$ \\
\hline & & 2.0 & 0.9464 & 0.9521 & 0.9554 & 0.9483 & 0.9515 & 0.9824 \\
\hline & & & (293.3686) & (3.9878) & $(4.0900)$ & (3.4771) & (3.5501) & (2.7229) \\
\hline \multirow[t]{8}{*}{100} & \multirow[t]{8}{*}{0.2} & 0.1 & 0.9461 & 0.9591 & $\mathbf{0 . 9 5 3 7}$ & 0.9434 & 0.9352 & 0.8726 \\
\hline & & & $(0.8134)$ & $(0.9378)$ & (0.9241) & $(0.9166)$ & $(0.9035)$ & $(0.6757)$ \\
\hline & & 0.5 & 0.9965 & 0.9691 & 0.9679 & 0.9508 & 0.9470 & 0.2665 \\
\hline & & & (3.4026) & (1.6493) & (1.6684) & (1.5542) & (1.5659) & $(0.3647)$ \\
\hline & & 1.0 & 0.9947 & 0.9613 & 0.9631 & 0.9433 & 0.9420 & 0.6284 \\
\hline & & & (13.0351) & (3.7143) & (3.8315) & (3.2441) & (3.3177) & (1.0735) \\
\hline & & 2.0 & 0.9326 & 0.9547 & 0.9601 & 0.9384 & 0.9410 & 0.8846 \\
\hline & & & (181.1185) & (16.6849) & $(17.6932)$ & (12.4128) & (12.9191) & (6.1118) \\
\hline
\end{tabular}


Table 1. Continued.

\begin{tabular}{lllllllll}
\hline \multirow{2}{*}{$n_{i}$} & \multirow{2}{*}{$\delta_{i}$} & \multirow{2}{*}{$\sigma_{i}^{2}$} & \multicolumn{6}{c}{ Coverage probabilities (Expected lengths) } \\
\cline { 4 - 8 } & & & FGCI & E-B.Indj & E-B.Uni & C-B.Indj & C-B.Uni & MOVER \\
\hline 100 & 0.5 & 0.1 & $\mathbf{0 . 9 6 8 5}$ & $\mathbf{0 . 9 5 7 8}$ & $\mathbf{0 . 9 5 8 5}$ & 0.9445 & 0.9462 & 0.8992 \\
& & $\mathbf{( 0 . 3 4 9 1 )}$ & $(0.3777)$ & $(0.3764)$ & $(0.3724)$ & $(0.3710)$ & $(0.2704)$ \\
\cline { 2 - 8 } & 0.5 & $\mathbf{0 . 9 9 9 6}$ & $\mathbf{0 . 9 6 0 4}$ & $\mathbf{0 . 9 6 3 2}$ & 0.9495 & $\mathbf{0 . 9 5 0 1}$ & 0.3157 \\
& & $(1.3697)$ & $(0.6091)$ & $(0.6121)$ & $(0.5933)$ & $(\mathbf{0 . 5 9 6 1})$ & $(0.1058)$ \\
\cline { 2 - 8 } & 1.0 & $\mathbf{0 . 9 9 9 0}$ & $\mathbf{0 . 9 5 5 3}$ & $\mathbf{0 . 9 5 7 4}$ & 0.9379 & 0.9415 & 0.7982 \\
& & $(3.7435)$ & $\mathbf{( 1 . 2 2 5 7 )}$ & $(1.2378)$ & $(1.1620)$ & $(1.1725)$ & $(0.4857)$ \\
\cline { 2 - 8 } & 2.0 & $\mathbf{0 . 9 6 6 8}$ & $\mathbf{0 . 9 5 4 5}$ & $\mathbf{0 . 9 5 4 8}$ & 0.9442 & 0.9475 & $\mathbf{0 . 9 5 4 9}$ \\
& & $(32.9162)$ & $(4.0100)$ & $(4.0691)$ & $(3.6039)$ & $(3.6477)$ & $\mathbf{( 2 . 4 3 4 3 )}$ \\
\hline 0.8 & 0.1 & $\mathbf{0 . 9 9 5 0}$ & $\mathbf{0 . 9 5 8 1}$ & $\mathbf{0 . 9 6 7 6}$ & 0.9450 & $\mathbf{0 . 9 5 5 9}$ & 0.5995 \\
& & $(0.2532)$ & $(0.2194)$ & $(0.2200)$ & $(0.2164)$ & $\mathbf{( 0 . 2 1 7 0 )}$ & $(0.1167)$ \\
\cline { 2 - 7 } & 0.5 & 1.0000 & $\mathbf{0 . 9 5 6 2}$ & $\mathbf{0 . 9 5 9 5}$ & 0.9465 & $\mathbf{0 . 9 5 0 3}$ & 0.7093 \\
& & $(1.0189)$ & $(0.3729)$ & $(0.3754)$ & $(0.3648)$ & $\mathbf{( 0 . 3 6 7 2 )}$ & $(0.1204)$ \\
\cline { 2 - 7 } & 1.0 & $\mathbf{0 . 9 9 9 2}$ & $\mathbf{0 . 9 5 3 7}$ & $\mathbf{0 . 9 5 6 2}$ & 0.9443 & 0.9465 & $\mathbf{0 . 9 5 6 0}$ \\
& & $(2.3875)$ & $(0.7571)$ & $(0.7631)$ & $(0.7289)$ & $(0.7344)$ & $\mathbf{( 0 . 4 7 9 6 )}$ \\
\cline { 2 - 7 } & 2.0 & $\mathbf{0 . 9 6 6 2}$ & 0.9496 & $\mathbf{0 . 9 5 2 2}$ & 0.9445 & 0.9479 & $\mathbf{0 . 9 8 7 2}$ \\
& & $(16.9898)$ & $(2.3221)$ & $(2.3448)$ & $(2.1655)$ & $(2.1851)$ & $(\mathbf{1 . 7 7 4 0})$ \\
\hline
\end{tabular}

Note: E-B.Indj and E-B.Uni represented the respective equal-tailed Bayesian intervals based on independent Jeffreys and uniform priors, and C-B.Indj and C-B.Uni represented the respective Bayesian credible intervals based on independent Jeffrey's and uniform priors. Bold denoted as the coverage probability $\geq 0.95$ and the shortest expected length.

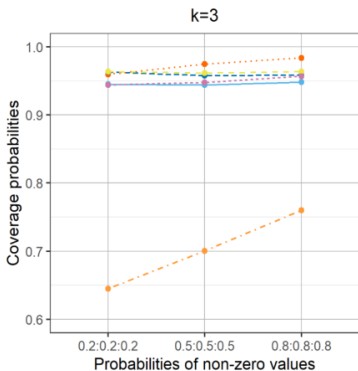

(A)

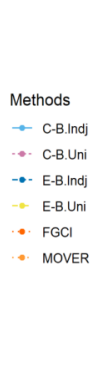

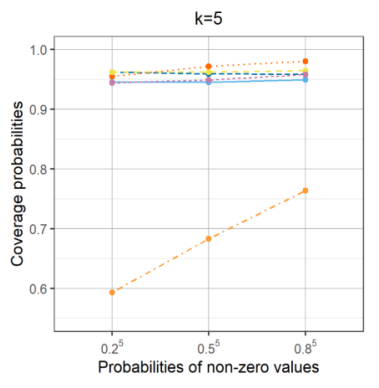

(B)

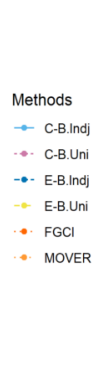

- C-B. Indj
- C-B.Uni
- E-B.Indj
- E-B.Uni
- FGCI
- MOVER
-

(C)

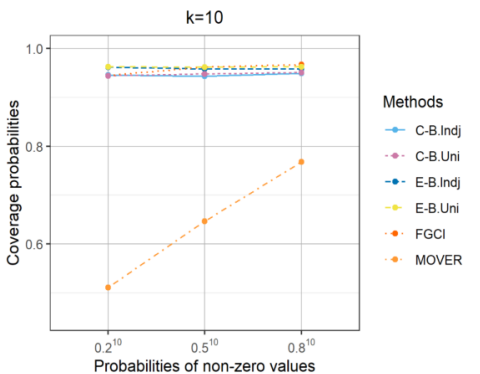

Figure 4. Comparison of the coverage probabilities of the proposed methods according to probabilities of non-zero values for (A) $k=3$ (B) $k=5$ (C) $k=10$.

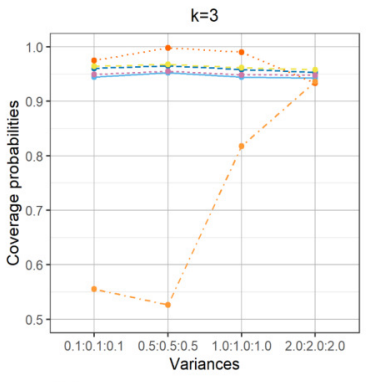

(A)

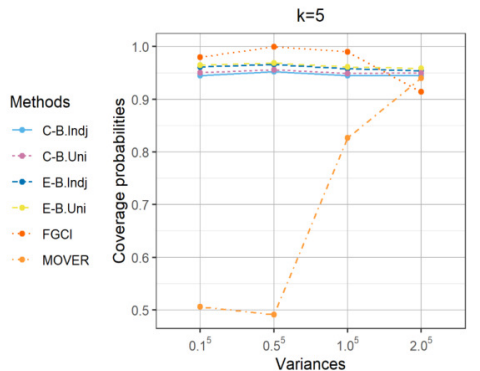

(B)

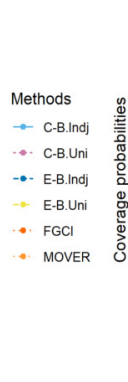

(C)

Figure 5. Comparison of the coverage probabilities of the proposed methods according to variances for (A) $k=3$ (B) $k=5$ (C) $k=10$. 
Table 2. The results for the $95 \%$ two-sided confidence intervals for the common CV of delta-lognormal distributions for $k=5$.

\begin{tabular}{|c|c|c|c|c|c|c|c|c|}
\hline \multirow{2}{*}{$n_{i}$} & \multirow{2}{*}{$\delta_{i}$} & \multirow{2}{*}{$\sigma_{i}^{2}$} & \multicolumn{6}{|c|}{ Coverage probabilities (Expected lengths) } \\
\hline & & & FGCI & E-B.Indj & E-B.Uni & C-B.Indj & C-B.Uni & MOVER \\
\hline \multirow[t]{15}{*}{25} & \multirow{8}{*}{0.5} & \multirow[t]{2}{*}{0.1} & $\mathbf{0 . 9 8 3 4}$ & 0.9596 & 0.9646 & 0.9374 & 0.9438 & 0.1197 \\
\hline & & & $(0.7505)$ & $(0.7303)$ & (0.7259) & $(0.7088)$ & $(0.7045)$ & $(0.3301)$ \\
\hline & & \multirow[t]{2}{*}{0.5} & 0.9997 & 0.9710 & 0.9746 & 0.9531 & 0.9595 & 0.4830 \\
\hline & & & $(3.7038)$ & (1.5339) & $(1.6273)$ & (1.3637) & $(1.4246)$ & $(0.3499)$ \\
\hline & & \multirow[t]{2}{*}{1.0} & 0.9737 & 0.9600 & 0.9647 & 0.9423 & 0.9506 & 0.7917 \\
\hline & & & (8.9088) & $(4.2573)$ & (4.7602) & (3.2717) & (3.5364) & (1.2034) \\
\hline & & \multirow[t]{2}{*}{2.0} & 0.8603 & 0.9541 & 0.9613 & 0.9410 & 0.9483 & 0.9096 \\
\hline & & & (1933.3646) & (32.2422) & $(40.4745)$ & (17.2785) & (19.8882) & (5.9389) \\
\hline & \multirow[t]{7}{*}{0.8} & \multirow[t]{2}{*}{0.1} & 0.9997 & 0.9697 & 0.9840 & 0.9485 & 0.9730 & 0.0701 \\
\hline & & & $(0.6148)$ & $(0.4309)$ & $(0.4382)$ & $(0.4187)$ & $(0.4265)$ & $(0.1438)$ \\
\hline & & \multirow[t]{2}{*}{0.5} & 1.0000 & 0.9639 & 0.9713 & 0.9574 & 0.9671 & 0.8460 \\
\hline & & & $(3.4724)$ & $(0.8808)$ & $(0.9159)$ & $(\mathbf{0 . 8 1 6 0})$ & $(0.8447)$ & $(0.3396)$ \\
\hline & & \multirow[t]{2}{*}{1.0} & 0.9836 & 0.9583 & 0.9637 & 0.9510 & 0.9614 & 0.9642 \\
\hline & & & $(10.7410)$ & $(2.0830)$ & $(2.1955)$ & (1.7916) & $(1.8719)$ & (1.0345) \\
\hline & & 2.0 & $\begin{array}{l}0.8901 \\
(3.67 \mathrm{E}+04)\end{array}$ & $\begin{array}{l}\mathbf{0 . 9 5 1 7} \\
(9.7354)\end{array}$ & $\begin{array}{l}\mathbf{0 . 9 5 7 6} \\
(10.5885)\end{array}$ & $\begin{array}{l}0.9493 \\
(7.0602)\end{array}$ & $\begin{array}{l}\mathbf{0 . 9 5 6 0} \\
(7.5199)\end{array}$ & $\begin{array}{l}\mathbf{0 . 9 8 0 7} \\
(4.1883)\end{array}$ \\
\hline \multirow[t]{24}{*}{50} & \multirow{8}{*}{0.2} & \multirow[t]{2}{*}{0.1} & 0.9532 & 0.9604 & 0.9526 & 0.9401 & 0.9310 & 0.4326 \\
\hline & & & (1.2400) & $(1.3288)$ & (1.3007) & (1.2798) & $(1.2516)$ & $(0.7301)$ \\
\hline & & \multirow[t]{2}{*}{0.5} & 0.9987 & 0.9743 & 0.9730 & 0.9556 & 0.9546 & 0.2693 \\
\hline & & & $(5.1024)$ & $(3.0276)$ & (3.2924) & (2.5680) & (2.7012) & $(0.5583)$ \\
\hline & & \multirow[t]{2}{*}{1.0} & 0.9647 & 0.9655 & 0.9690 & 0.9441 & 0.9467 & 0.6413 \\
\hline & & & $(16.6022)$ & (10.0192) & (12.3389) & $(6.7304)$ & $(7.6160)$ & $(1.6963)$ \\
\hline & & 2.0 & 0.8562 & 0.9539 & 0.9636 & 0.9407 & 0.9498 & 0.8377 \\
\hline & & & $(3.08 \mathrm{E}+05)$ & (187.9496) & (362.9737) & $(60.4077)$ & $(85.9882)$ & (10.2873) \\
\hline & 0.5 & 0.1 & 0.9803 & 0.9609 & 0.9630 & 0.9457 & 0.9501 & 0.7854 \\
\hline & & & $(0.5169)$ & $(0.5283)$ & $(0.5251)$ & $(0.5183)$ & $(0.5151)$ & $(0.3322)$ \\
\hline & & 0.5 & 1.0000 & 0.9661 & 0.9688 & 0.9519 & 0.9550 & 0.3610 \\
\hline & & & (2.4601) & $(0.9163)$ & $(0.9303)$ & $(0.8721)$ & $(0.8834)$ & $(0.1694)$ \\
\hline & & 1.0 & 0.9990 & 0.9582 & 0.9620 & 0.9446 & 0.9489 & 0.8114 \\
\hline & & & (6.0419) & $(2.0201)$ & $(2.0770)$ & (1.8123) & $(1.8540)$ & $(0.6894)$ \\
\hline & & 2.0 & 0.9276 & 0.9529 & 0.9570 & 0.9440 & 0.9463 & 0.9459 \\
\hline & & & (1419.0445) & (7.9109) & $(8.2496)$ & $(6.2988)$ & $(6.5140)$ & $(3.4257)$ \\
\hline & 0.8 & 0.1 & 0.9996 & 0.9640 & 0.9743 & 0.9492 & 0.9641 & 0.2056 \\
\hline & & & $(0.3905)$ & $(0.3067)$ & $(0.3087)$ & $(0.3012)$ & $(0.3032)$ & $(0.1238)$ \\
\hline & & 0.5 & 1.0000 & 0.9650 & 0.9703 & 0.9546 & 0.9618 & 0.8014 \\
\hline & & & (1.8753) & $(0.5542)$ & $(0.5624)$ & $(0.5337)$ & $(0.5412)$ & $(0.1868)$ \\
\hline & & 1.0 & 0.9993 & 0.9541 & 0.9547 & 0.9456 & 0.9501 & 0.9760 \\
\hline & & & $(4.2360)$ & (1.1716) & (1.1949) & (1.0922) & (1.1119) & $(0.6724)$ \\
\hline & & 2.0 & 0.9321 & 0.9552 & 0.9576 & 0.9518 & 0.9555 & 0.9932 \\
\hline & & & (45.8789) & $(4.0252)$ & (4.1253) & (3.5054) & (3.5809) & $(2.5923)$ \\
\hline 100 & 0.2 & 0.1 & 0.9507 & 0.9586 & 0.9514 & 0.9442 & 0.9368 & 0.9115 \\
\hline & & & $(0.8347)$ & $(0.9364)$ & $(0.9220)$ & $(0.9150)$ & $(0.9014)$ & $(0.6683)$ \\
\hline & & 0.5 & 0.9995 & 0.9667 & 0.9640 & 0.9484 & 0.9459 & 0.1510 \\
\hline & & & (3.6099) & (1.6424) & (1.6607) & $(1.5490)$ & $(1.5595)$ & $(0.2957)$ \\
\hline & & 1.0 & 0.9980 & 0.9626 & 0.9638 & 0.9438 & 0.9443 & 0.6174 \\
\hline & & & (8.7078) & (3.7265) & (3.8501) & (3.2539) & (3.3293) & $(0.8938)$ \\
\hline & & 2.0 & 0.9178 & 0.9578 & 0.9612 & 0.9435 & 0.9464 & 0.8842 \\
\hline & & & (1302.6130 & (16.4958) & (17.4678) & (12.2904) & (12.7883) & $(5.0720)$ \\
\hline
\end{tabular}


Table 2. Continued.

\begin{tabular}{|c|c|c|c|c|c|c|c|c|}
\hline \multirow{2}{*}{$n_{i}$} & \multirow{2}{*}{$\delta_{i}$} & \multirow{2}{*}{$\sigma_{i}^{2}$} & \multicolumn{6}{|c|}{ Coverage probabilities (Expected lengths) } \\
\hline & & & FGCI & E-B.Indj & E-B.Uni & C-B.Indj & C-B.Uni & MOVER \\
\hline \multirow[t]{16}{*}{100} & \multirow[t]{8}{*}{0.5} & 0.1 & 0.9707 & 0.9559 & 0.9573 & 0.9452 & 0.9448 & 0.9455 \\
\hline & & & $(\mathbf{0 . 3 5 9 8})$ & $(0.3784)$ & $(0.3768)$ & $(0.3730)$ & $(0.3715)$ & $(0.2692)$ \\
\hline & & 0.5 & 1.0000 & 0.9618 & 0.9621 & 0.9494 & 0.9494 & 0.2473 \\
\hline & & & (1.5439) & (0.6091) & $(0.6120)$ & $(0.5933)$ & (0.5958) & $(0.0838)$ \\
\hline & & 1.0 & 1.0000 & 0.9541 & 0.9577 & 0.9425 & 0.9459 & 0.8244 \\
\hline & & & (3.9566) & (1.2291) & $(1.2421)$ & $(1.1653)$ & $(1.1763)$ & $(0.4456)$ \\
\hline & & 2.0 & 0.9672 & 0.9541 & 0.9558 & 0.9444 & 0.9464 & 0.9717 \\
\hline & & & $(15.8155)$ & $(4.0068)$ & $(4.0614)$ & $(3.6001)$ & (3.6428) & (2.2992) \\
\hline & \multirow[t]{8}{*}{0.8} & 0.1 & 0.9985 & 0.9622 & 0.9688 & 0.9499 & 0.9598 & 0.5759 \\
\hline & & & $(0.2646)$ & $(0.2194)$ & $(0.2200)$ & $(0.2164)$ & $(0.2171)$ & $(0.1142)$ \\
\hline & & 0.5 & 1.0000 & 0.9607 & 0.9647 & 0.9483 & 0.9549 & 0.7699 \\
\hline & & & $(1.1485)$ & $(0.3738)$ & $(0.3762)$ & $(0.3656)$ & $(0.3679)$ & $(0.1127)$ \\
\hline & & 1.0 & 1.0000 & 0.9499 & $\mathbf{0 . 9 5 3 0}$ & 0.9430 & 0.9461 & $\mathbf{0 . 9 8 5 7}$ \\
\hline & & & $(2.6290)$ & $(0.7549)$ & $(0.7615)$ & $(0.7270)$ & $(0.7329)$ & $(0.4680)$ \\
\hline & & 2.0 & 0.9598 & 0.9516 & 0.9523 & 0.9466 & 0.9489 & 0.9969 \\
\hline & & & $(6.1687)$ & $(2.3383)$ & $(2.3604)$ & $(2.1803)$ & (2.1988) & (1.7304) \\
\hline
\end{tabular}

Note: Bold denoted as the coverage probability $\geq 0.95$ and the shortest expected length.

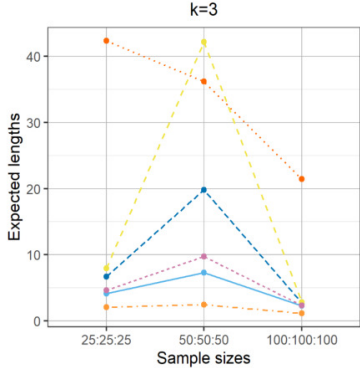

(A)

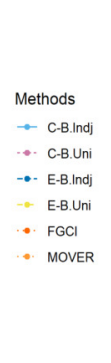

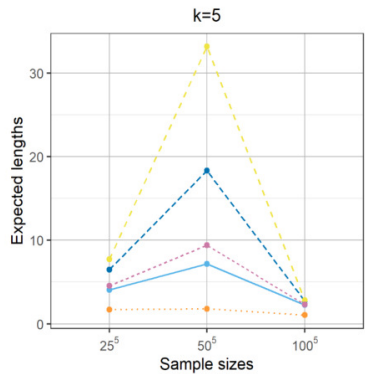

(B)

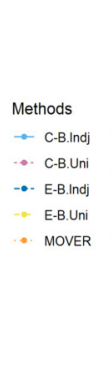

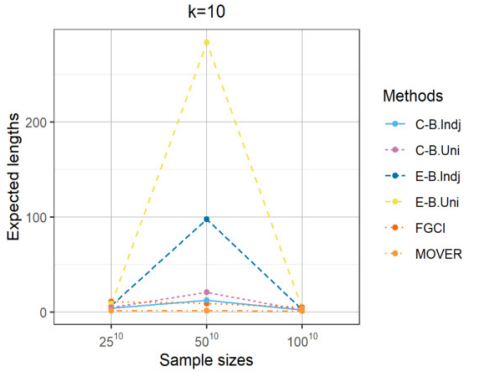

(C)

Figure 6. Comparison of the expected lengths of the proposed methods according to sample sizes for (A) $k=3$ (B) $k=5$ (C) $k=10$.

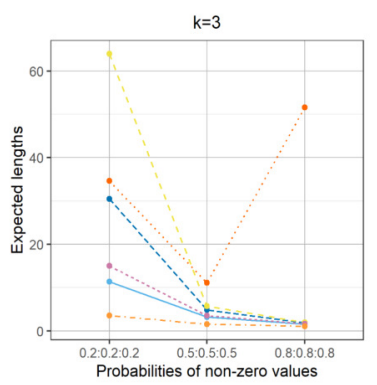

(A)

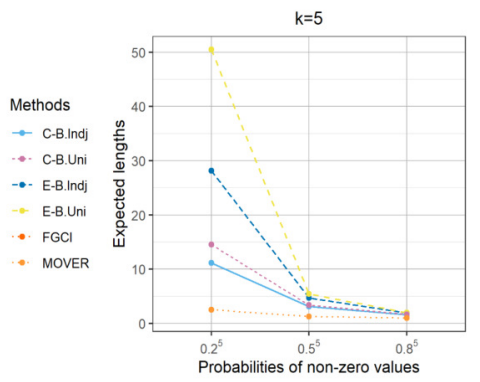

(B)

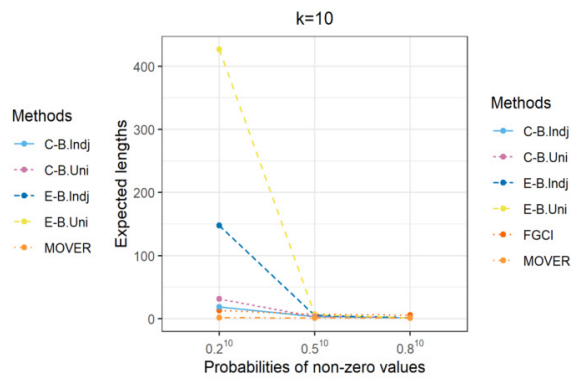

(C)

Figure 7. Comparison of the expected lengths of the proposed methods according to probabilities of non-zero values for (A) $k=3$ (B) $k=5$ (C) $k=10$.

discussed previously. AIC and BIC were used to test the possible distributions of these datasets in which the non-zero observations follow a right-skewed distribution (Tables 4 and 5, respectively). The results indicate that the non-zero observations in the three datasets most closely follow a lognormal distribution. 
Table 3. The results for the $95 \%$ two-sided confidence intervals for the common CV of delta-lognormal distributions for $k=10$.

\begin{tabular}{|c|c|c|c|c|c|c|c|c|}
\hline \multirow[b]{2}{*}{$n_{i}$} & \multirow[b]{2}{*}{$\delta_{i}$} & \multirow{2}{*}{$\sigma_{i}^{2}$} & \multicolumn{6}{|c|}{ Coverage probabilities (Expected lengths) } \\
\hline & & & FGCI & E-B.Indj & E-B.Uni & C-B.Indj & C-B.Uni & MOVER \\
\hline \multirow[t]{16}{*}{25} & \multirow[t]{8}{*}{0.5} & 0.1 & 0.9750 & 0.9578 & 0.9648 & 0.9353 & 0.9420 & 0.0262 \\
\hline & & & $(0.7387)$ & $(0.7284)$ & $(0.7247)$ & $(0.7070)$ & $(0.7032)$ & $(0.3056)$ \\
\hline & & 0.5 & 0.9999 & 0.9682 & 0.9716 & 0.9500 & 0.9550 & 0.3282 \\
\hline & & & (3.7907) & $(1.5242)$ & $(1.6165)$ & (1.3565) & $(1.4164)$ & $(0.2387)$ \\
\hline & & 1.0 & 0.9721 & 0.9568 & 0.9646 & 0.9396 & 0.9486 & 0.7670 \\
\hline & & & (7.9902) & (4.2284) & $(4.7050)$ & (3.2447) & $(3.5022)$ & $(0.9681)$ \\
\hline & & 2.0 & 0.8170 & 0.9544 & 0.9617 & 0.9399 & 0.9486 & 0.9124 \\
\hline & & & $(27.2845)$ & (35.0401) & (46.9413) & (18.2674) & $(21.6147)$ & (4.5799) \\
\hline & \multirow[t]{8}{*}{0.8} & 0.1 & 1.0000 & 0.9695 & 0.9829 & 0.9511 & 0.9734 & 0.0118 \\
\hline & & & $(0.5753)$ & $(0.4324)$ & $(0.4395)$ & $(0.4201)$ & $(0.4275)$ & $(0.1331)$ \\
\hline & & 0.5 & 1.0000 & 0.9658 & 0.9731 & 0.9566 & 0.9677 & 0.9021 \\
\hline & & & $(3.4758)$ & $(0.8807)$ & $(0.9150)$ & $(\mathbf{0 . 8 1 5 5 )}$ & $(0.8441)$ & $(0.3032)$ \\
\hline & & 1.0 & 0.9796 & 0.9538 & 0.9594 & 0.9494 & 0.9584 & 0.9804 \\
\hline & & & (7.0148) & $(2.0894)$ & (2.2045) & (1.7966) & (1.8782) & $(\mathbf{0 . 9 3 3 5})$ \\
\hline & & 2.0 & 0.8306 & 0.9552 & 0.9601 & 0.9488 & 0.9557 & 0.9900 \\
\hline & & & $(37.3845)$ & (9.6166) & (10.4834) & (6.9970) & (7.4504) & (3.6911) \\
\hline \multirow[t]{24}{*}{50} & \multirow[t]{8}{*}{0.2} & 0.1 & 0.9490 & 0.9603 & 0.9553 & 0.9430 & 0.9356 & 0.2560 \\
\hline & & & $(1.2475)$ & (1.3345) & (1.3065) & (1.2848) & (1.2569) & (0.6806) \\
\hline & & 0.5 & 0.9979 & 0.9723 & 0.9724 & 0.9541 & 0.9518 & 0.1051 \\
\hline & & & (5.3392) & (3.0554) & (3.3326) & (2.5815) & (2.7157) & (0.3897) \\
\hline & & 1.0 & 0.9650 & 0.9636 & 0.9685 & 0.9442 & 0.9463 & 0.5255 \\
\hline & & & (11.0842) & (10.4725) & (12.9382) & (6.9200) & (7.8607) & (1.1454) \\
\hline & & 2.0 & 0.8124 & 0.9575 & 0.9658 & 0.9442 & 0.9522 & 0.8009 \\
\hline & & & $(53.4270)$ & (1142.6720) & (3369.2176) & (122.0329) & $(222.4721)$ & (6.6526) \\
\hline & \multirow[t]{8}{*}{0.5} & 0.1 & 0.9700 & 0.9601 & 0.9632 & 0.9453 & 0.9495 & 0.7897 \\
\hline & & & $(0.5115)$ & $(0.5278)$ & $(0.5248)$ & $(0.5177)$ & $(0.5149)$ & $(0.3240)$ \\
\hline & & 0.5 & 1.0000 & 0.9672 & 0.9702 & 0.9516 & 0.9553 & 0.2112 \\
\hline & & & (2.6159) & $(0.9159)$ & $(0.9302)$ & $(0.8720)$ & $(0.8828)$ & (0.1163) \\
\hline & & 1.0 & 0.9970 & 0.9596 & 0.9621 & 0.9458 & 0.9507 & 0.8171 \\
\hline & & & $(6.1760)$ & (2.0213) & (2.0769) & (1.8134) & (1.8535) & (0.6006) \\
\hline & & 2.0 & 0.8956 & 0.9540 & 0.9584 & 0.9415 & 0.9459 & 0.9652 \\
\hline & & & (15.6125) & (8.0203) & (8.3825) & (6.3810) & (6.5924) & (3.0276) \\
\hline & \multirow[t]{8}{*}{0.8} & 0.1 & 0.9976 & 0.9652 & 0.9745 & 0.9507 & 0.9651 & 0.0741 \\
\hline & & & $(0.3688)$ & $(0.3067)$ & $(0.3088)$ & $(0.3012)$ & $(0.3033)$ & $(0.1153)$ \\
\hline & & 0.5 & 0.9596 & 0.9557 & 0.9487 & 0.9433 & 0.8688 & 0.9300 \\
\hline & & & (2.0073) & $(0.5524)$ & $(0.5606)$ & $(0.5322)$ & $(0.5395)$ & $(0.1726)$ \\
\hline & & 1.0 & 0.9975 & 0.9554 & 0.9598 & 0.9451 & 0.9516 & 0.9910 \\
\hline & & & (4.2894) & $(1.1691)$ & (1.1913) & 99) & (1.1089) & $(0.6371)$ \\
\hline & & 2.0 & 0.8936 & 0.9554 & 0.9579 & 0.9513 & 0.9566 & 0.9992 \\
\hline & & & (7.8557) & (4.0458) & (4.1465) & (3.5224) & $(3.5975)$ & (2.4638) \\
\hline \multirow[t]{8}{*}{100} & \multirow[t]{8}{*}{0.2} & 0.1 & 0.9445 & 0.9577 & 0.9534 & 0.9437 & 0.9349 & 0.9551 \\
\hline & & & $(0.8343)$ & $(0.9358)$ & $(0.9215)$ & $(0.9147)$ & $(0.9008)$ & $(0.6605)$ \\
\hline & & 0.5 & 0.9996 & 0.9680 & 0.9669 & 0.9550 & 0.9487 & 0.0384 \\
\hline & & & (3.8651) & (1.6467) & (1.6645) & (1.5521) & (1.5622) & $(0.2242)$ \\
\hline & & 1.0 & 0.9942 & 0.9614 & 0.9623 & 0.9459 & 0.9445 & 0.5322 \\
\hline & & & (8.9324) & (3.7215) & (3.8417) & (3.2516) & (3.3245) & $(0.6967)$ \\
\hline & & 2.0 & 0.8966 & 0.9539 & 0.9579 & 0.9389 & 0.9412 & 0.8751 \\
\hline & & & $(22.0831)$ & (16.7659) & (17.8464) & (12.4326) & (12.9750) & (4.1159) \\
\hline
\end{tabular}


Table 3. Continued.

\begin{tabular}{lllllllll}
\hline \multirow{2}{*}{$n_{i}$} & \multirow{2}{*}{$\delta_{i}$} & \multirow{2}{*}{$\sigma_{i}^{2}$} & \multicolumn{5}{c}{ Coverage probabilities (Expected lengths) } \\
\cline { 4 - 8 } 100 & 0.5 & 0.1 & $\mathbf{0 . 9 6 1 2}$ & $\mathbf{0 . 9 5 4 7}$ & $\mathbf{0 . 9 5 6 2}$ & 0.9431 & 0.9439 & $\mathbf{0 . 9 8 1 6}$ \\
& & $(0.3580)$ & $(0.3777)$ & $(0.3764)$ & $(0.3724)$ & $(0.3711)$ & $(\mathbf{0 . 2 6 8 0})$ \\
\cline { 2 - 8 } & 0.5 & 1.0000 & $\mathbf{0 . 9 6 2 3}$ & $\mathbf{0 . 9 6 2 4}$ & $\mathbf{0 . 9 5 1 2}$ & $\mathbf{0 . 9 5 1 9}$ & 0.1266 \\
& & $(1.6621)$ & $(0.6085)$ & $(0.6115)$ & $\mathbf{( 0 . 5 9 2 7})$ & $(0.5954)$ & $(0.0594)$ \\
\cline { 2 - 8 } & 1.0 & 1.0000 & $\mathbf{0 . 9 5 6 1}$ & $\mathbf{0 . 9 5 8 2}$ & 0.9433 & 0.9462 & 0.8441 \\
& & $(4.1570)$ & $\mathbf{( 1 . 2 3 0 3 )}$ & $(1.2424)$ & $(1.1663)$ & $(1.1766)$ & $(0.4028)$ \\
\cline { 2 - 8 } & 2.0 & $\mathbf{0 . 9 5 6 5}$ & 0.9497 & $\mathbf{0 . 9 5 2 3}$ & 0.9404 & 0.9409 & $\mathbf{0 . 9 8 9 9}$ \\
& & $(8.2323)$ & $(4.0172)$ & $(4.0756)$ & $(3.6098)$ & $(3.6542)$ & $(\mathbf{2 . 1 7 9 5 )}$ \\
\hline 0.8 & 0.1 & $\mathbf{0 . 9 9 2 3}$ & $\mathbf{0 . 9 5 9 8}$ & $\mathbf{0 . 9 6 7 9}$ & 0.9494 & $\mathbf{0 . 9 5 8 9}$ & 0.5202 \\
& & $(0.2599)$ & $(0.2192)$ & $(0.2198)$ & $(0.2163)$ & $\mathbf{( 0 . 2 1 6 8 )}$ & $(0.1112)$ \\
\cline { 2 - 7 } & 0.5 & 1.0000 & $\mathbf{0 . 9 5 9 0}$ & $\mathbf{0 . 9 6 3 1}$ & $\mathbf{0 . 9 5 0 1}$ & $\mathbf{0 . 9 5 4 7}$ & 0.8216 \\
& & $(1.2451)$ & $(0.3745)$ & $(0.3770)$ & $\mathbf{( 0 . 3 6 6 3 )}$ & $(0.3686)$ & $(0.1055)$ \\
\cline { 2 - 7 } & 1.0 & 1.0000 & $\mathbf{0 . 9 5 5 1}$ & $\mathbf{0 . 9 5 6 8}$ & 0.9476 & 0.9483 & $\mathbf{0 . 9 9 7 5}$ \\
& & $(2.7509)$ & $(0.7554)$ & $(0.7609)$ & $(0.7273)$ & $(0.7324)$ & $\mathbf{( 0 . 4 5 8 2})$ \\
\cline { 2 - 7 } & 2.0 & $\mathbf{0 . 9 5 1 8}$ & 0.9497 & $\mathbf{0 . 9 5 2 6}$ & 0.9469 & 0.9480 & $\mathbf{0 . 9 9 9 9}$ \\
& & $(4.0077)$ & $(2.3383)$ & $(2.3610)$ & $(2.1800)$ & $(2.1998)$ & $(\mathbf{1 . 6 9 5 5})$ \\
\hline
\end{tabular}

Note: Bold denoted as the coverage probability $\geq 0.95$ and the shortest expected length.

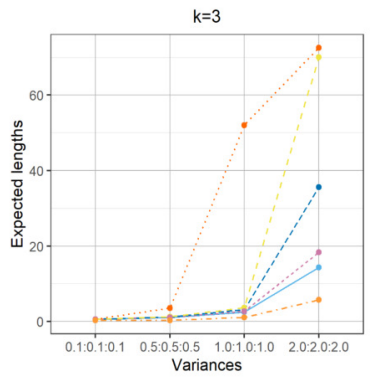

(A)
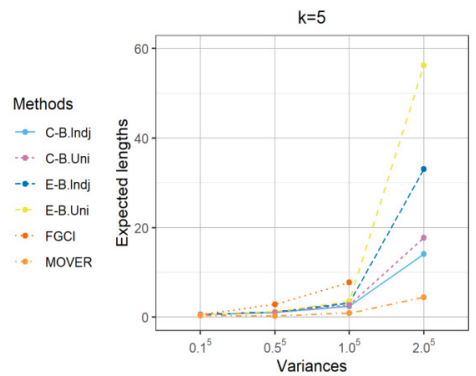

(B)

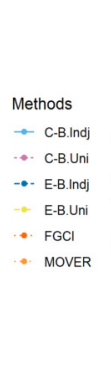

-B.ind

E-B.Indj

E-B.Uni

MOVEe

(1)

$(\mathrm{C})$

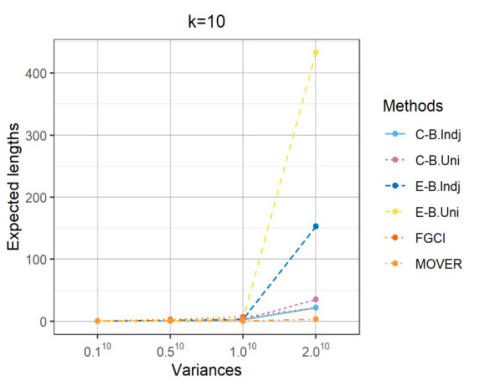

(C)

Figure 8. Comparison of the expected lengths of the proposed methods according to variances for (A) $k=3(\mathrm{~B}) k=5(\mathrm{C}) k=10$.

Furthermore, the normal Q-Q plots via the log-transformation of non-zero observations shown in Fig 2 reveal that they follow normal distributions. By testing the non-zero observations together with the binomial distributions of the true zero observations indicate that the daily rainfall data from the three areas follow delta-lognormal distributions.

The summary statistics for the three daily rainfall datasets were $n_{1}: n_{2}: n_{3}=62: 62: 62 ; \hat{\delta}_{1}: \hat{\delta}_{2}: \hat{\delta}_{3}=$ $0.7258: 0.7903: 0.7419 ; \hat{\mu}_{1}: \hat{\mu}_{2}: \hat{\mu}_{3}=2.1189: 1.6448: 1.8971 ; \hat{\sigma}_{1}^{2}: \hat{\sigma}_{2}^{2}: \hat{\sigma}_{3}^{2}=1.7857: 3.4406: 1.8346$; and $\tilde{\eta}=3.2011$. The $95 \%$ confidence and credible intervals for the common CV of the three daily rainfall datasets are summarized in Table 6 . The results reveal that the three confidence intervals tested contained the real value of the parameter, thereby reinforcing the conclusions based on the simulation study results. However, the expected length of FGCI was the shortest, thereby making it a good choice for estimating the common CV in the dispersion of precipitation from the three areas in Nan province, Thailand.

\section{DISCUSSION}

We extended the idea of Thangjai et al. (2020b) who established confidence intervals using FGCI for the common $\mathrm{CV}$ of lognormal distributions to the context of the same distribution but with excess zeros. We then applied it to examine the dispersion in three daily rainfall datasets. In our case, the findings from the simulation study infer that the Bayesian methods were preferable to FGCI and MOVER for 
Table 4. The AIC values of the non-zero observations from Chiang Klang, Tha Wang Pha, and Pua in Nan, Thailand.

\begin{tabular}{lllllll}
\hline \multirow{2}{*}{ Areas } & \multicolumn{2}{l}{ Distributions } & & & & \\
\cline { 2 - 7 } & Normal & Lognormal & Cauchy & Exponential & Gamma & Weibull \\
\hline Chiang Klang & 430.7372 & $\mathbf{3 4 7 . 4 8 3 5}$ & 387.5394 & 356.1853 & 355.0152 & 353.0018 \\
\hline Tha Wang Pha & 477.9087 & $\mathbf{3 6 3 . 7 7 8 5}$ & 415.4823 & 386.6203 & 366.9576 & 363.8529 \\
\hline Pua & 425.2069 & $\mathbf{3 3 5 . 9 7 6 0}$ & 379.7269 & 346.6206 & 344.5560 & 342.1551 \\
\hline
\end{tabular}

Note: Bold denoted as the minimum AIC.

Table 5. The BIC values of the non-zero observations from Chiang Klang, Tha Wang Pha, and Pua in Nan, Thailand.

\begin{tabular}{lllllll}
\hline \multirow{2}{*}{ Areas } & \multicolumn{2}{l}{ Distributions } & & & & \\
\cline { 2 - 7 } & Normal & Lognormal & Cauchy & Exponential & Gamma & Weibull \\
\hline Chiang Klang & 434.3506 & $\mathbf{3 5 1 . 0 9 6 8}$ & 391.1527 & 357.9920 & 358.6286 & 356.6151 \\
\hline Tha Wang Pha & 481.6923 & $\mathbf{3 6 7 . 5 6 2 1}$ & 419.2659 & 388.5121 & 370.7412 & 367.6365 \\
\hline Pua & 428.8642 & $\mathbf{3 3 9 . 6 3 3 3}$ & 383.3842 & 348.4493 & 348.2133 & 345.8124 \\
\hline
\end{tabular}

Note: Bold denoted as the minimum BIC.

Table 6. The $95 \%$ confidence intervals and credible intervals for the common CV of daily rainfall datasets from Chiang Klang, Tha Wang Pha, and Pua in Nan, Thailand.

\begin{tabular}{llll}
\hline Methods & Lower & Upper & Lengths \\
\hline FGCI & 2.0363 & 4.4528 & 2.4165 \\
\hline E-B.Indj & 1.8975 & 4.7920 & 2.8945 \\
\hline E-B.U & 1.9039 & 5.0631 & 3.1592 \\
\hline C-B.Indj & 1.7583 & 4.3644 & 2.6061 \\
\hline C-B.U & 1.7540 & 4.4703 & 2.7163 \\
\hline MOVER & 2.3642 & 5.1399 & 2.7757 \\
\hline
\end{tabular}

almost all cases. Although MOVER performed well for cases with a high proportion of non-zero values, it produced coverage probabilities that were lower than the nominal confidence level for cases with small variances. This is probably because the lower and upper bounds of the zero values are used in the confidence interval construction, and the combined effect with the other parameters caused the inadequate coverage probability results.

Note that, a referee suggested to add additional simulation with small discrepancy to the probability distribution and evaluate coverage under such conditions. We added noise additive with $\mu_{i}=0$ and set $\sigma_{i}^{2}$ to four conditions: $0.1,0.5,1.0$, and by Tables $1-3$. We found that for the additional noise additive with $\mu_{i}=0$ and $\sigma_{i}^{2}=0.1$, the coverage probabilities and the expected lengths (not shown here) are similar to those in Tables 1-3, indicating that the coverage performance of the simulation results in the manuscript does not deteriorate. In the case of the additional noise additive with $\mu_{i}=0$ and $\sigma_{i}^{2}=0.5$, the performance of the coverage slightly deteriorates. In addition, when $\sigma_{i}^{2}$ increases for the remaining conditions, the performance of the coverage is substantially deteriorated. Finally, in this paper we focus on the coverage probability of proposed confidence intervals of delta lognormal distribution in (1) only. For the referee's valuable comments, we shall find new confidence intervals which has the coverage probability at least the nominal level 0.95 , when $\sigma_{i}^{2}$ is increasing more than 0.5 in the next study.

\section{CONCLUSION}

Confidence intervals for the common $\mathrm{CV}$ of delta-lognormal distribution were constructed based on FGCI, two equal-tailed Bayesian credible intervals using the independent Jeffreys or uniform priors, and MOVER. Their coverage probabilities and expected lengths under various simulation scenarios were used 
to assess their efficacies. The equal-tailed Bayesian credible interval based on the independent Jeffreys prior provided superior coverage probabilities compared to other methods. Moreover, the equal-tailed Bayesian based on the uniform prior can be used as an alternative. This is due to the parameter to be estimated relying on the posterior densities of $\delta_{i}^{*}$ and $\sigma_{i}^{2}$. According to the results of the simulation study and the real data example, the Bayesian credible interval based on the independent Jeffreys prior is suitable for cases with small variances since it provided the narrowest length of the interval due to it falling in the domain of its posterior density. Furthermore, MOVER is the best choice when the proportion of non-zero values is high and the variance is large.

\section{REFERENCES}

Aitchison, J. (1955). On the distribution of a positive random variable having a discrete probability mass at the origin. Journal of the American Statistical Association, 50(271):901-908.

Behboodian, J. and Jafari, A. A. (2008). Generalized confidence interval for the common coefficient of variation. Journal of Statistical Theory and Applications, 7(3):349-363.

Bolstad, W. M. and Curran, J. M. (2016). Introduction to Bayesian statistics. John Wiley \& Sons, Hoboken, New Jersey, 3rd edition.

Chen, Y.-H. and Zhou, X.-H. (2006). Generalized confidence intervals for the ratio or difference of two means for lognormal populations with zeros. UW Biostatistics Working Paper Series, page Working Paper 296.

DasGupta, A. (2008). Asymptotic theory of statistics and probability. Springer Texts in Statistics. Springer-Verlag, New York.

Fletcher, D. (2008). Confidence intervals for the mean of the delta-lognormal distribution. Environmental and Ecological Statistics, 15(2):175-189.

Fukuchi, H. (1988). Correlation properties of rainfall rates in the United Kingdom. IEE Proceedings $H$ (Microwaves, Antennas and Propagation), 135(2):83-88.

Gupta, R. C., Ramakrishnan, S., and Zhou, X. (1999). Point and interval estimation of $\mathrm{P}(\mathrm{X}<\mathrm{Y})$ : the normal case with common coefficient of variation. Annals of the Institute of Statistical Mathematics, 51(3):571-584.

Hannig, J. (2009). On generalized fiducial inference. Statistica Sinica, 19(2):491-544.

Hannig, J., Iyer, H., and Patterson, P. (2006). Fiducial generalized confidence intervals. Journal of the American Statistical Association, 101(473):254-269.

Harvey, J. and van der Merwe, A. J. (2012). Bayesian confidence intervals for means and variances of lognormal and bivariate lognormal distributions. Journal of Statistical Planning and Inference, 142(6):1294-1309.

Hasan, M. S. and Krishnamoorthy, K. (2018). Confidence intervals for the mean and a percentile based on zero-inflated lognormal data. Journal of Statistical Computation and Simulation, 88(8):1499-1514.

Hayter, A. J. (2015). Confidence bounds on the coefficient of variation of a normal distribution with applications to win-probabilities. Journal of Statistical Computation and Simulation, 85(18):37783791.

Kong, C. Y., Jamaludin, S., Yusof, F., and Foo, H. M. (2012). Parameter estimation for bivariate mixed lognormal distribution. Journal of Science and Technology, 4(1):41-48.

Krishnamoorthy, K. and Mathew, T. (2003). Inferences on the means of lognormal distributions using generalized p-values and generalized confidence intervals. Journal of Statistical Planning and Inference, 115(1):103-121.

Krishnamoorthy, K. and Oral, E. (2017). Standardized likelihood ratio test for comparing several lognormal means and confidence interval for the common mean. Statistical Methods in Medical Research, 26(6):2919-2937.

Kvanli, A. H., Shen, Y. K., and Deng, L. Y. (1998). Construction of confidence intervals for the mean of a population containing many zero values. Journal of Business \& Economic Statistics, 16(3):362-368.

Li, X., Zhou, X., and Tian, L. (2013). Interval estimation for the mean of lognormal data with excess zeros. Statistics \& Probability Letters, 83(11):2447-2453.

Liu, X. and Xu, X. (2015). A note on combined inference on the common coefficient of variation using confidence distributions. Electronic Journal of Statistics, 9(1):219-233.

Maneerat, P., Niwitpong, S.-A., and Niwitpong, S. (2018). Confidence intervals for the ratio of means of delta-lognormal distribution. In Anh, L. H., Dong, L. S., Kreinovich, V., and Thach, N. N., editors, 
Econometrics for Financial Applications, volume 760 of Studies in Computational Intelligence, pages 161-174. Springer International Publishing, Cham.

Maneerat, P., Niwitpong, S.-A., and Niwitpong, S. (2019a). Bayesian confidence intervals for a single mean and the difference between two means of delta-lognormal distributions. Communications in Statistics - Simulation and Computation, pages 1-29.

Maneerat, P., Niwitpong, S.-A., and Niwitpong, S. (2019b). Confidence intervals for the mean of deltalognormal distribution. In Kreinovich, V. and Sriboonchitta, S., editors, Structural Changes and their Econometric Modeling, volume 808 of Studies in Computational Intelligence, pages 264-274. Springer International Publishing, Cham.

Maneerat, P., Niwitpong, S.-A., and Niwitpong, S. (2020a). A Bayesian approach to construct confidence intervals for comparing the rainfall dispersion in Thailand. PeerJ, 8:e8502.

Maneerat, P., Niwitpong, S.-A., and Niwitpong, S. (2020b). Bayesian confidence intervals for the difference between variances of delta-lognormal distributions. Biometrical Journal, 62(7):1769-1790.

Nam, J.-M. and Kwon, D. (2017). Inference on the ratio of two coefficients of variation of two lognormal distributions. Communications in Statistics - Theory and Methods, 46(17):8575-8587.

NASA (2020). Climate change evidence: how do we know? Available at https://climate.nasa.gov/evidence. (accessed 10 September 2020).

Nema, P., Nema, S., and Roy, P. (2012). An overview of global climate changing in current scenario and mitigation action. Renewable and Sustainable Energy Reviews, 16(4):2329-2336.

$\mathrm{Ng}$, C. K. (2014). Inference on the common coefficient of variation when populations are lognormal: a simulation-based approach. Journal of Statistics: Advances in Theory and Applications, 11(2):117-134.

O'Reilly, J. X. and Mars, R. B. (2015). Bayesian models in cognitive neuroscience: a tutorial. In Forstmann, B. U. and Wagenmakers, E.-J., editors, An Introduction to Model-Based Cognitive Neuroscience, pages 179-197. Springer, New York, NY.

Pang, W.-K., Leung, P.-K., Huang, W.-K., and Liu, W. (2005). On interval estimation of the coefficient of variation for the three-parameter Weibull, lognormal and gamma distribution: A simulation-based approach. European Journal of Operational Research, 164(2):367-377.

Shimizu, K. (1993). A bivariate mixed lognormal distribution with an analysis of rainfall data. Journal of Applied Meteorology and Climatology, 32(2):161-171.

Stone, J. V. (2013). Bayes' rule: tutorial introduction to Bayesian analysis. Sebtel Press, Sheffield.

Thai Meteorological Department (2015). The climate of Thailand. Available at https://www.tmd.go.th/en/archive/ thailand_climate.pdf. (accessed 30 August 2020).

Thangjai, W. and Niwitpong, S.-A. (2017). Confidence intervals for the weighted coefficients of variation of two-parameter exponential distributions. Cogent Mathematics, 4(1):1315880.

Thangjai, W., Niwitpong, S.-A., and Niwitpong, S. (2020a). Adjusted generalized confidence intervals for the common coefficient of variation of several normal populations. Communications in Statistics Simulation and Computation, 49(1):194-206.

Thangjai, W., Niwitpong, S.-A., and Niwitpong, S. (2020b). Confidence intervals for the common coefficient of variation of rainfall in Thailand. PeerJ, 8:e10004.

Tian, L. (2005). Inferences on the mean of zero-inflated lognormal data: the generalized variable approach. Statistics in Medicine, 24(20):3223-3232.

Tian, L. and Wu, J. (2006). Confidence intervals for the mean of lognormal data with excess zeros. Biometrical Journal. Biometrische Zeitschrift, 48(1):149-156.

Weerahandi, S. (1993). Generalized confidence intervals. Journal of the American Statistical Association, 88(423):899-905.

WorldAtlas (2021). Southeast Asia. Available at https://www.worldatlas.com/geography/southeastasia.html. (accessed 4 July 2021).

Wu, W.-H. and Hsieh, H.-N. (2014). Generalized confidence interval estimation for the mean of deltalognormal distribution: an application to New Zealand trawl survey data. Journal of Applied Statistics, 41(7):1471-1485.

Yosboonruang, N. and Niwitpong, S. (2020). Statistical inference on the ratio of delta-lognormal coefficients of variation. Applied Science and Engineering Progress.

Yosboonruang, N., Niwitpong, S., and Niwitpong, S.-A. (2019a). Confidence intervals for coefficient of variation of three parameters delta-lognormal distribution. In Kreinovich, V. and Sriboonchitta, S., 
editors, Structural Changes and their Econometric Modeling, volume 808 of Studies in Computational Intelligence, pages 352-363. Springer International Publishing, Cham.

Yosboonruang, N., Niwitpong, S.-A., and Niwitpong, S. (2018). Confidence intervals for the coefficient of variation of the delta-lognormal distribution. In Anh, L. H., Dong, L. S., Kreinovich, V., and Thach, N. N., editors, Econometrics for Financial Applications, volume 760 of Studies in Computational Intelligence, pages 327-337. Springer International Publishing, Cham.

Yosboonruang, N., Niwitpong, S.-A., and Niwitpong, S. (2019b). Measuring the dispersion of rainfall using Bayesian confidence intervals for coefficient of variation of delta-lognormal distribution: a study from Thailand. PeerJ, 7:e7344.

Yosboonruang, N., Niwitpong, S.-A., and Niwitpong, S. (2020). The Bayesian confidence intervals for measuring the difference between dispersions of rainfall in Thailand. PeerJ, 8:e9662.

Yue, S. (2000). The bivariate lognormal distribution to model a multivariate flood episode. Hydrological Processes, 14(14):2575-2588.

Zhou, X. H. and Tu, W. (2000). Confidence intervals for the mean of diagnostic test charge data containing zeros. Biometrics, 56(4):1118-1125.

Zou, G. Y. and Donner, A. (2008). Construction of confidence limits about effect measures: a general approach. Statistics in Medicine, 27(10):1693-1702. 